\title{
Adaptive Control Allocation
}

\author{
Johannes Tjønnås and Tor A. Johansen
}

Department of Engineering Cybernetics, Norwegian University of Science and Technology, Trondheim, Norway

\begin{abstract}
In this work we address the control allocation problem for a nonlinear over-actuated time-varying system where parameters affine in the effector model may be assumed unknown. Instead of optimizing the control allocation at each time instant, a dynamic approach is considered by constructing update-laws that represent asymptotically optimal allocation search and adaptation. Using Lyapunov analysis for cascaded set-stable systems, uniform global/local asymptotic stability is guaranteed for the sets described by the system, the optimal allocation update-law and the adaptive update-law.
\end{abstract}

Key words: Control allocation; Adaptive control; Nonlinear systems; Cascaded systems; Optimization.

\section{Introduction}

This work is motivated by the over-actuated control allocation problem. The problem is described by a nonlinear system, divided into a dynamic and a static part. The main contribution of this work is to show that the instantaneous control allocation problem, optimizing the desired input of the dynamic part of the system, not necessarily needs to be solved exactly at each time instant. In order to imply convergence and stability properties of the whole system, stability and attractivity of the optimal set, described by the control allocation problem, is pursued.

Optimizing control allocation solutions have been derived for certain classes of over-actuated systems, such as aircraft, automotive vehicles and marine vessels, (Enns 1998, Buffington, Enns \& Teel 1998, Sørdalen 1997, Bodson 2002, Härkegård 2002, Luo, Serrani, Yurkovich, Doman \& Oppenheimer 2004, Luo, Serrani, Yurkovich, Doman \& Oppenheimer 2005, Poonamallee, Yurkovich, Serrani, Doman \& Oppenheimer 2005, Johansen, Fossen \& Berge 2004) and (Johansen, Fossen \& Tøndel 2005). The control allocation problem is, in (Enns 1998, Buffington et al. 1998, Sørdalen 1997, Johansen et al. 2005, Bodson 2002) and (Härkegård 2002), viewed as a static or quasi-dynamic optimization problem that is solved independently of the dynamic control problem considering non-adaptive linear effector models of the form $\tau=G u$. In (Luo et al. 2004) and (Luo et al. 2005) a dynamic model predictive approach is considered to solve the allocation problem with linear time-varying dynamic in the actuator/effector model, $T \dot{u}+u=u_{c}$. In (Poonamallee et al. 2005) and (Johansen et al. 2004) sequential quadratic programming tech- niques are used to cope with nonlinearities in the control allocation problem due to singularity avoidance and actuator failure. The main advantage of the control allocation approach is in general modularity and the ability to handle redundancy and constraints.

Consider the system

$$
\begin{aligned}
& \dot{x}=f_{x}(t, x)+g_{x}(t, x) \tau \\
& \tau=\Phi(t, x, u, \theta):=\Phi_{\theta}(t, x, u) \theta+\Phi_{0}(t, x, u)
\end{aligned}
$$

where $t \geq 0, x \in \mathbb{R}^{n}$ is the state vector, $u \in \mathbb{R}^{r}$ is control input vector, $\tau \in \mathbb{R}^{d}$ is a vector of virtual controls and $\theta \in \mathbb{R}^{m}$ is a constant vector that contains parameters that either will be assumed known, or viewed as uncertain parameters to be adapted. The state $x$, is assumed known and typically represents the tracking error. The mapping (2) represents an actuator and effector model, where the control signals $u$, are mapped to the virtual controls $\tau$. We limit our study to the over-actuated control allocation problem, where $m \leq d \leq r$.

Problem: Assume that there exist a virtual control $\tau_{c}:=k(t, x)$, with $k(t, 0)=0$, that is differentiable and uniformly globally asymptotically stabilizes the origin of (1), when $\tau=k(t, x)$.

i) If $\theta$ is known, define an update law $\dot{u}:=f_{u}(t, x, u)$, such that the stability property of the closed loop is conserved and $u(t)$ converges to an optimal solution with respect to the static minimization problem

$$
\min _{u} J(t, x, u) \quad \text { s.t } \quad \tau_{c}-\Phi(t, x, u, \theta)=0 .
$$

ii) If $\theta$ is unknown, solve problem i) with $\theta=\hat{\theta}, \dot{u}:=$ $f_{u}(t, x, u, \hat{\theta})$ and define an adaptive update law $\dot{\hat{\theta}}:=$ $f_{\hat{\theta}}(t, x, u, \hat{\theta})$, where $\hat{\theta} \in \mathbb{R}^{m}$ is an estimate of $\theta$. 
In (Johansen 2004) it was shown that it is not necessary to solve the optimization problem (3) exactly at each time instant. Further a control Lyapunov function was used to derive an exponentially convergent updatelaw for $u$ (similar to a gradient or Newton-like optimization) such that the control allocation problem (3) could be solved dynamically. And finally it was shown that convergence and asymptotic optimality of the system, composed by the dynamic control allocation and a uniform globally exponentially stable trajectory-tracking controller, guarantees uniform boundedness and uniform global exponential convergence to the optimal solution of the system. The advantage of this approach is computational efficiency and simplicity of implementation, since the optimizing control allocation algorithm is implemented as a dynamic nonlinear controller. Solving (3) online at each sampling instant requires a computationally more expensive numerical solution. In (Tjønnås \& Johansen 2005) the results from (Johansen 2004) were extended by allowing uncertain parameters, associated with an adaptive law, in the effector model, and by applying set-stability analysis in order to also conclude asymptotic stability of the optimal solution set.

In what follows we will extend the ideas from (Johansen 2004) and (Tjønnås \& Johansen 2005) by utilizing the set-stability result for cascaded systems established in (Tjønnås, Chaillet, Panteley \& Johansen 2006). This result enables us to relax the assumptions in (Johansen 2004) and (Tjønnås \& Johansen 2005) where $f_{x}(t, x), g_{x}(t, x)$ and $\Phi(t, x, u, \theta)$ are assumed to be globally Lipschitz in $x$. Further the virtual controller $\tau_{c}$ does only need to render equilibrium of (1) UGAS, not UGES as assumed in (Johansen 2004) and (Tjønnås \& Johansen 2005). The implementation of the adaptive law presented in (Tjønnås \& Johansen 2005) depends directly on the Lyapunov function used in the analysis. In this work the analysis and implementation are separated, and although the Lyapunov functions in the analysis need to satisfy certain requirements, the adaptive implementation does not assume knowledge of these Lyapunov functions.

\section{Notation, definitions and preliminary results}

$|\cdot|$ denotes the Euclidian norm and $|\cdot|_{\mathcal{A}^{\prime}}: \mathbb{R}^{q} \rightarrow \mathbb{R}_{\geq 0}$ denotes the distance from a point $z \in \mathbb{R}^{q}$ to a set $\mathcal{A}^{\prime} \subset \mathbb{R}^{q},|z|_{\mathcal{A}^{\prime}}:=\inf \left\{|z-y|: y \in \mathcal{A}^{\prime}\right\}$. The solution of an autonomous dynamic system $\dot{z}=F(z)$ is denoted by $z\left(t, z_{0}\right)$ at $t \geq t_{0}$, where $z_{0}=z\left(t_{0}, z_{0}\right)$ is the initial state and $t_{0}$ is the initial time.

\subsection{Definitions}

The definitions that follows are either motivated by, or can be found in (Teel, Panteley \& Loria 2002) and (Lin, Sontag \& Wang 1996). They pertain to systems of the form

$$
\dot{z}=F(z) \text {, }
$$

where $z:=\left(p, x^{\mathrm{T}}\right)^{\mathrm{T}}, p$ is the time-state

$$
\dot{p}=1, \quad p_{0}=t_{0} \text {. }
$$

and $F: \mathbb{D} \rightarrow \mathbb{R}^{q}$ is locally Lipschitz with $\mathbb{D} \subset \mathbb{R}^{q}$. In the following, if referred to a set, it has the properties of being nonempty.

Definition 1 The system (4) is said to be forward complete if, for each $z_{0} \in \mathbb{D}$, the solution $z\left(\cdot, z_{0}\right) \in \mathbb{D}$ is defined on $\mathbb{R}_{\geq t_{0}}$.

Definition 2 The system (4) is said to be finite escape time detectable through $|\cdot|_{\mathcal{A}}$, if any solution, $z\left(t, z_{0}\right) \in \mathbb{D}$, which is right maximally defined on a bounded interval $\left[t_{0}, T\right)$, satisfies $\lim _{t \nearrow T}\left|z\left(t, z_{0}\right)\right|_{\mathcal{A}}=\infty$.

Definition 3 If the system (4) is forward complete, then the closed set $\mathcal{A} \subset \mathbb{D}$ is:

- Uniformly Stable (US), if there exists a function, $\nu \in$ $\mathcal{K}$, and a constant, $c>0$, such that, $\forall\left|z_{0}\right|_{\mathcal{A}}<c$,

$$
\left|z\left(t, z_{0}\right)\right|_{\mathcal{A}} \leq \nu\left(\left|z_{0}\right|_{\mathcal{A}}\right), \forall t \geq 0 .
$$

- Uniformly Globally Stable $(U G S)$, when $\mathbb{D}=\mathbb{R}^{q}$, if (6) is satisfied with, $\nu \in \mathcal{K}_{\infty}$, and for any $z_{0} \in \mathbb{R}^{q}$.

- Uniformly Attractive (UA) if there exists a constant $c>0$ such that for all $\left|z_{0}\right|_{\mathcal{A}}<c$ and any $\mu>0$ there exists $T=T(\mu)>0$, such that

$$
\left|z_{0}\right|_{\mathcal{A}} \leq c, t \geq T \Rightarrow\left|z\left(t, z_{0}\right)\right|_{\mathcal{A}} \leq \mu,
$$

- Uniformly Globally Attractive (UGA), when $\mathbb{D}=\mathbb{R}^{q}$, if for each pair of strictly positive numbers $(c, \mu)$ there exists $T=T(\mu)>0$ such that for all $z_{0} \in \mathbb{R}^{q}$, (7) holds.

- Uniformly Asymptotically Stable (UAS) if it is US and $U A$.

- Uniformly Globally Asymptotically Stable (UGAS), when $\mathbb{D}=\mathbb{R}^{q}$, if it is $U G S$ and $U G A$.

From (Khalil 1996), we adapt the definition of uniform boundedness of solutions to the case when $\mathcal{A}$ is not reduced to $\{0\}$.

Definition 4 With respect to the closed set $\mathcal{A} \subset \mathbb{R}^{q}$, the solutions of system (4) are said to be:

- Uniformly Bounded (UB) if there exist a positive constant $c$, such that for every positive constant $r<c$ there is a positive constant $\mu=\mu(r)$, such that

$$
\left|z_{0}\right|_{\mathcal{A}} \leq r \Rightarrow\left|z\left(t, z_{0}\right)\right|_{\mathcal{A}} \leq \mu, \quad \forall t \geq 0 .
$$

- Uniformly Globally Bounded (UGB), when $\mathbb{D}=\mathbb{R}^{q}$, if for every $r \in \mathbb{R}_{\geq 0}$, there is a positive constant $\mu=\mu(r)$ such that (8) is satisfied.

Definition 5 A smooth Lyapunov function for (4) with respect to a non-empty, closed forward invariant set $\mathcal{A} \subset \mathbb{D}$ is a function $V: \mathbb{D} \rightarrow \mathbb{R}$ that satisfies: $i)$ there exists two $\mathcal{K}$ functions $\alpha_{1}$ and $\alpha_{2}$ such that for any $z \in \mathbb{D}$, $\alpha_{1}\left(|z|_{\mathcal{A}}\right) \leq V(z) \leq \alpha_{2}\left(|z|_{\mathcal{A}}\right)$. ii) There exists a continuous and a positive definite or semidefinite function $\alpha_{3}$ such that for any $z \in \mathbb{D} \backslash \mathcal{A}: \frac{\partial V}{\partial z}(z) F(z) \leq-\alpha_{3}\left(|z|_{\mathcal{A}}\right)$.

\subsection{Preliminary results}

In order to conclude stability of a set of points, rather than a single equilibrium point, we have the following result from (Skjetne 2005). 
Theorem 1 Assume that the system (4) is finite escapetime detectable through $|z|_{\mathcal{A}}$. If there exists a smooth Lyapunov function for the system (4) with respect to a nonempty, closed, forward invariant set $\mathcal{A}$, then $\mathcal{A}$ is $U G S$ with respect to (4). Furthermore, if $\alpha_{3}$ is a positive definite function, then $\mathcal{A}$ is UGAS with respect to (4), and if $\alpha_{i}\left(|z|_{\mathcal{A}}\right)=c_{i}|z|_{\mathcal{A}}^{r}$ for $i=1,2,3$ where $c_{i}$ and $r$ are strictly positive values and $r>1$, then $\mathcal{A}$ is $U G E S$ with respect to (4).

Consider the cascaded system

$$
\begin{aligned}
& \dot{x}_{1}=f_{c 1}\left(t, x_{1}\right)+g_{c}\left(t, x_{1}, x_{2}\right) \\
& \dot{x}_{2}=f_{c 2}\left(t, x_{2}\right) .
\end{aligned}
$$

When the functions $f_{c 1}, f_{c 2}$ and $g_{c}$ are locally Lipschitz in all arguments, this class of nonlinear time-varying systems can be represented by the following autonomous system

$$
\begin{aligned}
& \dot{z}_{1}=F_{1}\left(z_{1}\right)+G(z) \\
& \dot{z}_{2}=F_{2}\left(z_{2}\right)
\end{aligned}
$$

where $z_{1}:=\left(p, x_{1}^{\mathrm{T}}\right)^{\mathrm{T}} \in \mathbb{R}^{q_{1}}, z_{2}:=\left(p, x_{2}^{\mathrm{T}}\right)^{\mathrm{T}} \in \mathbb{R}^{q_{2}}$, $z:=\left(p, x^{\mathrm{T}}\right)^{\mathrm{T}} \in \mathbb{R}^{q}, x:=\left(x_{1}^{\mathrm{T}}, x_{2}^{\mathrm{T}}\right)^{\mathrm{T}}, F_{1}\left(z_{1}\right):=$ $\left(1, f_{c 1}\left(p, x_{1}\right)^{\mathrm{T}}\right)^{\mathrm{T}}, G(z):=\left(0, g_{c}(p, x)^{\mathrm{T}}\right)^{\mathrm{T}}$ and $F_{2}\left(z_{2}\right):=$ $\left(1, f_{c 2}\left(p, x_{2}\right)^{\mathrm{T}}\right)^{\mathrm{T}}$. Based on the cascaded system formulation we will make use of the results from (Tjønnås et al. 2006).

Lemma 1 Let $\mathcal{O}_{1}$ and $\mathcal{O}_{2}$ be some closed subsets of $\mathbb{R}^{q_{1}}$ and $\mathbb{R}^{q_{2}}$ respectively. Then, under the following assumptions, the set $\mathcal{A}:=\mathcal{O}_{1} \times \mathcal{O}_{2}$ is UGAS with respect to the cascade (11)-(12).

A 1 The set $\mathcal{O}_{2}$ is UGAS with respect to the system (12) and that the solution of the system (11)-(12) is UGB with respect to $\mathcal{A}$.

$\boldsymbol{A} 2$ The functions $F_{1}, F_{2}$ and $G$ are locally Lipschitz.

A 3 The cascade (11)-(12) is forward complete.

$\boldsymbol{A} 4$ There exist a continuous function $G_{1}: \mathbb{R}_{\geq 0} \rightarrow \mathbb{R}_{\geq 0}$ and a class $\mathcal{K}$ function $G_{2}$ such that, for all $z \in \mathbb{R}^{q}$,

$$
|G(z)| \leq G_{1}\left(|z|_{\mathcal{A}}\right) G_{2}\left(\left|z_{2}\right|_{\mathcal{O}_{2}}\right) \text {. }
$$

$\boldsymbol{A} \boldsymbol{5}$ There exists a continuously differentiable function $\bar{V}_{1}: \mathbb{R}^{q_{1}} \rightarrow \mathbb{R}_{\geq 0}$, class $\mathcal{K}_{\infty}$ functions $\bar{\alpha}_{1}, \bar{\alpha}_{2}$ and $\bar{\alpha}_{3}$, and a continuous function $\bar{\varsigma}_{1}: \mathbb{R}_{\geq 0} \rightarrow \mathbb{R}_{\geq 0}$ such that, for all $z_{1} \in \mathbb{R}^{q_{1}}$

$$
\begin{aligned}
\bar{\alpha}_{1}\left(\left|z_{1}\right|_{\mathcal{O}_{1}}\right) & \leq \bar{V}_{1}\left(z_{1}\right) \leq \bar{\alpha}_{2}\left(\left|z_{1}\right|_{\mathcal{O}_{1}}\right) \\
\frac{\partial \bar{V}_{1}}{\partial z_{1}}\left(z_{1}\right) F_{1}\left(z_{1}\right) & \leq-\bar{\alpha}_{3}\left(\left|z_{1}\right|_{\mathcal{O}_{1}}\right) \\
\left|\frac{\partial \bar{V}_{1}}{\partial x_{1}}\left(z_{1}\right)\right| & \leq \bar{\varsigma}\left(\left|z_{1}\right|_{\mathcal{O}_{1}}\right) .
\end{aligned}
$$

Corollary 1 Let $\mathcal{O}_{1}$ and $\mathcal{O}_{2} \subset \mathbb{D}$ be closed subsets of $\mathbb{R}^{q_{1}}$ and $\mathbb{R}^{q_{2}}$ respectively, and the assumptions $A 2-A 5$ be satisfied. Then, with respect to the cascade (11)-(12), the set $\mathcal{A}$ is:
- $U G S$, when $\mathbb{D}=\mathbb{R}^{q_{2}}$, if $\mathcal{O}_{2}$ is $U G S$ with respect to the system (12) and that the solution of system (11)-(12) is $U G B$ with respect to $\mathcal{A}$.

- UAS, if $\mathrm{O}_{2}$ is $U A S$ with respect to the system (12).

- US, if $\mathrm{O}_{2}$ is US with respect to the system (12).

\section{Optimizing adaptive control allocation}

The adaptive control allocation approach is based on three modular steps, see also Figure 1.

(1) The high level control algorithm. The virtual control $\tau$ is treated as an available input to system (1), and a virtual control law $\tau_{c}:=k(t, x)$ is designed such that the origin of (1) is UGAS.

(2) The control allocation algorithm. Based on the minimization problem (3) where $J$ is a cost function that incorporates objectives such as minimum power consumption and actuator constraints (implemented as barrier functions), the Lagrangian function

$L(t, x, u, \lambda)=J(t, x, u)+(k(t, x)-\Phi(t, x, u, \theta))^{\mathrm{T}} \lambda$

is introduced. Update laws for the control input $u$ and the Lagrangian parameter $\lambda$ are then defined such that $u$ and $\lambda$ converges to a set defined by the time-varying optimality condition.

(3) The adaptive algorithm. In order to cope with an unknown parameter vector $\theta$ in the effector model, an adaptive law is defined. The parameter estimate is used in the control allocation algorithm and a certainty equivalent adaptive optimal control allocation can be defined.

High level control law
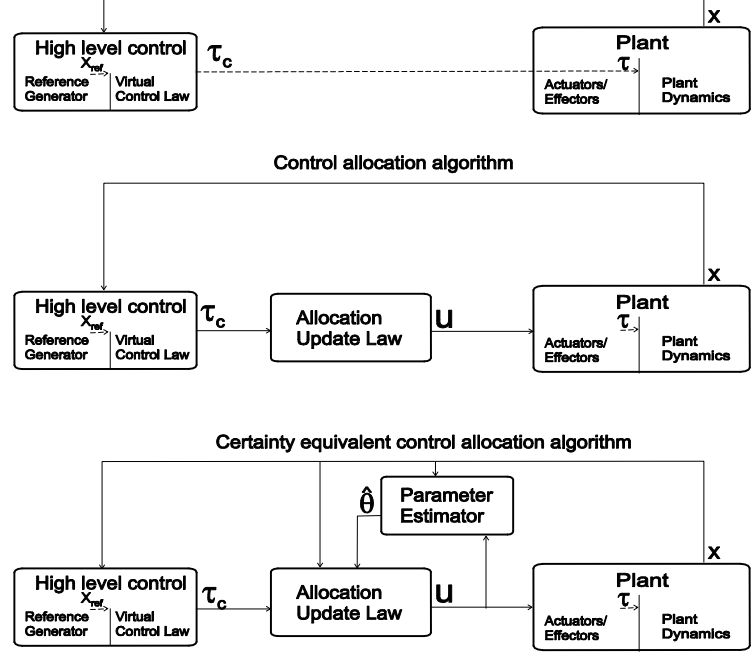

Fig. 1. Adaptive control allocation design philosophy 


\subsection{High level control algorithm}

In this section we state the main system assumptions and clarify the requirements on the high level control algorithm.

Assumption 1 (Plant)

a) There exists a continuous function $G_{f}: \mathbb{R}_{\geq 0} \rightarrow \mathbb{R}_{>0}$ and a constant $K$, such that $\left|f_{x}(t, x)\right| \leq G_{f}(|x|)$ and $\left|g_{x}(t, x)\right| \leq K$ for all $t$ and $x$. Moreover $f_{x}$ is locally Lipschitz in $t$ and $x$, and $f_{x}(t, 0)=0$.

b) There exist a continuous function $\varsigma_{\partial g_{x}}: \mathbb{R}_{\geq 0} \rightarrow \mathbb{R}_{\geq 0}$ such that for allt and $x, g_{x}$ is differentiable and $\left|\frac{\partial g_{x}}{\partial t}\right|+$ $\left|\frac{\partial g_{x}}{\partial x}\right| \leq \varsigma_{\partial g_{x}}(|x|)$.

c) The function $\Phi$ is twice differentiable and there exist a continuous function $G_{\Phi}: \mathbb{R}_{>0} \rightarrow \mathbb{R}_{\geq 0}$, such that $\left|\Phi_{\theta}\right|+\left|\Phi_{0}\right| \leq G_{\Phi}\left(\left|\left(x^{T}, u^{T}\right)^{T}\right|\right)$ for all $t, x$, and $u$. Further there exist a continuous function $\varsigma_{\partial \Phi}(|x|) \geq$ $\left|\frac{\partial \Phi_{\theta}}{\partial t}\right|+\left|\frac{\partial \Phi_{0}}{\partial t}\right|+\left|\frac{\partial \Phi_{\theta}}{\partial x}\right|+\left|\frac{\partial \Phi_{0}}{\partial x}\right|+\left|\frac{\partial \Phi_{\theta}}{\partial u}\right|+\left|\frac{\partial \Phi_{0}}{\partial u}\right|$ where $\varsigma_{\partial \Phi}: \mathbb{R}_{>0} \rightarrow \mathbb{R}_{\geq 0}$.

d) There exists constants $\varrho_{2}>\varrho_{1}>0$, such that $\forall t, x$, $u$ and $\theta$

$$
\varrho_{1} I<\frac{\partial \Phi}{\partial u}(t, x, u, \theta)\left(\frac{\partial \Phi}{\partial u}(t, x, u, \theta)\right)^{T} \leq \varrho_{2} I .
$$

Remark 1 Assumption 1 d) can be viewed as a controllability assumption in the sense that: i) the mapping $\Phi(t, x, \cdot, \theta): \mathbb{R}^{r} \rightarrow \mathbb{R}^{d}$ is surjective for all $t, x$ and $\theta$ and ii) for all $t, x$ and $\theta$ there exist a continuous function $f_{u}(t, x, \theta)$ such that $\Phi\left(t, x, f_{u}(t, x, \theta), \theta\right)=k(t, x)$. The surjective property can be seen by the Moore-Penrose pseudoinverse solution, $u_{1}=\left.\frac{\partial \Phi}{\partial u}\right|_{u_{c}} ^{T}\left(\left.\left.\frac{\partial \Phi}{\partial u}\right|_{u_{c}} \frac{\partial \Phi}{\partial u}\right|_{u_{c}} ^{T}\right)^{-1} y$, of the equation, $y=\left.\frac{\partial \Phi}{\partial u}\right|_{u_{c}} u_{1}$, which exist for any $u_{c}$. Thus for every $y$ there exists a solution $\Phi\left(t, x, u_{1}, \theta\right)=$ $\Phi(t, x, 0, \theta)+y$ by the Mean Value Theorem, where $u_{c} \in$ $\left(0, u_{1}\right)$. ii) follows from the Implicit Function Theorem by $i)$ and Assumption $1 \mathrm{~d}$ ).

\section{Assumption 2 (High Level Control Algorithm)}

a) There exists a feedback control $\tau_{c}:=k(t, x) \leq \varsigma_{k}(|x|)$, where $\varsigma_{k}: \mathbb{R}_{>0} \mapsto \mathbb{R}_{>0}$ is a continuous function, that render the equilibrium of (1) UGAS for $\tau=\tau_{c}$. The function $k$ is differentiable, $\left|\frac{\partial k}{\partial t}\right|+\left|\frac{\partial k}{\partial x}\right| \leq \varsigma_{\partial k}(|x|)$, where $\varsigma_{\partial k}: \mathbb{R}_{\geq 0} \mapsto \mathbb{R}_{\geq 0}$ is continuous, and $k(t, 0)=0$ for all $t$.

If we rewrite (1) such that

$$
\dot{x}=f(t, x)+g(t, x)(k(t, x)-\Phi(t, x, u, \theta))
$$

where $f(t, x):=f_{x}(t, x)+g_{x}(t, x) k(t, x), g(t, x):=$ $-g_{x}(t, x)$, by Assumption 1 and 2 the functions $f$ and $g$ have the same properties as $f_{x}$ and $g_{x}$. Also the equilibrium of

$$
\dot{x}=f(t, x) \text {, }
$$

the set

$$
\mathcal{O}_{x}:=\left\{x \in \mathbb{R}^{n} \mid x=0\right\},
$$

is UGAS by Assumption 2.

From Assumption 2 a) there exists a Lyapunov function $V_{x}: \mathbb{R}_{>0} \times \mathbb{R}^{n} \rightarrow \mathbb{R}_{>0}$ and $\mathcal{K}_{\infty}$ functions $\alpha_{x 1}, \alpha_{x 2}, \alpha_{x 3}$ and $\alpha_{x 4}$ such that

$$
\begin{aligned}
\alpha_{x 1}(|x|) & \leq V_{x}(t, x) \leq \alpha_{x 2}(|x|) \\
\frac{\partial V_{x}}{\partial t}+\frac{\partial V_{x}}{\partial x} f(t, x) & \leq-\alpha_{x 3}(|x|) \\
\left|\frac{\partial V_{x}}{\partial x}\right| & \leq \alpha_{x 4}(|x|)
\end{aligned}
$$

for the system $\dot{x}=f(t, x)$ with respect to its origin.

\section{Assumption 2 (Continued)}

b) There exists a $\mathcal{K}_{\infty}$ function $\alpha_{k}: \mathbb{R}_{\geq 0} \rightarrow \mathbb{R}_{\geq 0}$, such that

$$
\alpha_{x 4}(|x|) \alpha_{k}(|x|) \leq \alpha_{x 3}(|x|)
$$

Remark 2 If the origin of $\dot{x}=f(t, x)$ is UGES, then Assumption 2 is generally satisfied, with for example $\alpha_{x 3}$ quadratic, $\alpha_{x 4}$ linear and $\alpha_{k}$ sublinear, and $V_{x}$ does not need to be known explicitly in order to verify Assumption $2 b)$.

\subsection{Certainty equivalent adaptive control allocation al- gorithm}

In this section we first establish update-laws for the control input $u$, the Lagrangian multipliers $\lambda$, and the parameter estimate $\hat{\theta}$, such that stability and convergence with respect to the time-varying first order optimal set defined by the optimization problem (3) can be concluded. Then we show that the stabilizing properties of the virtual controller from Assumption 2, are conserved for the closed loop system.

In order to account for the unknown, but bounded, parameter vector $\theta$, an adaptive mechanism is include in the optimal control allocation design. We use a Lyapunov based indirect parameter estimation scheme (see for example (Krstic, Kanellakopoulos \& Kokotovic 1995) for a systematic Lyapunov based procedure). Since the state vector of (1) is assumed to be known, we consider the estimation model:

$$
\dot{\hat{x}}=A(x-\hat{x})+f_{x}(t, x)+g_{x}(t, x) \Phi(t, x, u, \hat{\theta})
$$

in the construction of the adaptive law. This estimation model has the same structure as a typical series parallel model (SP) (Ioannou \& Sun 1996), (Landau 1979). For analytical purpose, the filtered error estimate of the unknown parameter vector:

$$
\dot{\epsilon}=-A \epsilon+g_{x}(t, x) \Phi_{\theta}(t, x, u) \tilde{\theta},
$$

where $\tilde{\theta}=\theta-\hat{\theta}, \epsilon=x-\hat{x}$ and $(-A)$ is Hurwitz, will be used. 
The analysis and design of the certainty equivalent adaptive control allocation algorithm can be carried out in several ways. We consider the approach, with reference to Figure 2, where the perturbing system $\left(\Sigma_{2}\right)$ is defined by the adaptive law and the allocation algorithm dynamics, while the perturbed system $\left(\Sigma_{1}\right)$ is defined by the system dynamics. An advantage with this approach

\section{Cascaded System Interpretation}

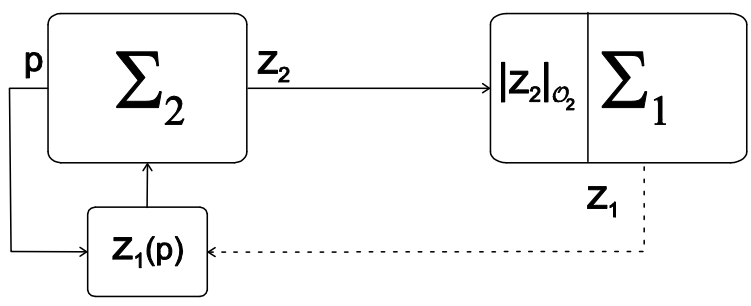

Fig. 2. General representation of a time-variant cascade, where $\Sigma_{1}$ is the perturbed system that will be UGAS with respect to a set, $\mathcal{O}_{1}$, when $\left|z_{2}\right|_{\mathcal{O}_{2}}=0 . \Sigma_{2}$ is the perturbing system. Note that $\Sigma_{2}$ may be perturbed indirectly by $\Sigma_{1}$ since $z_{1}$ may be considered as a time-varying signal, $z_{1}(t)$, as long as this signal exists for all $t$.

is that, the adaptive law is independent of $V_{x}$.

Remark 3 A different approach would be to expand the perturbed system $\left(\Sigma_{1}\right)$ with an adaptive law. In this case the adaptive law will be dependent on the initial Lyapunov function (similar to (Tjønnås \& Johansen 2005)), but convergence results like, $x(t) \rightarrow 0$ as $t \rightarrow \infty$, may be concluded even if a persistence of excitation condition is not satisfied.

\subsubsection{Stability of the optimal certainty equivalent con- trol allocation}

In order to see the cascaded coupling between the system (19) and the adaptive and optimal control allocation update-laws, equation (19) can be rewritten by:

$$
\begin{aligned}
\dot{x}= & f(t, x)+g(t, x)(k(t, x)-\Phi(t, x, u, \hat{\theta})) \\
& +g_{x}(t, x) \Phi_{\theta}(t, x, u) \tilde{\theta} .
\end{aligned}
$$

Based on the perturbing system, we consider the estimated optimal solution set:

$\mathcal{O}_{u \lambda \tilde{\theta}}(t, x):=\left\{(u, \lambda, \epsilon \tilde{\theta}) \in \mathbb{R}^{n} u \lambda \tilde{\theta} \mid f_{\mathcal{O}_{u \lambda \tilde{\theta}}}\left(t, z_{x u \lambda \tilde{\theta}}\right)=0\right\}$

where $n_{u \lambda \tilde{\theta}}:=r+d+n+m, z_{x u \lambda \tilde{\theta}}:=(x, u, \lambda, \epsilon, \tilde{\theta})$, $f_{\mathcal{O}_{u \lambda \tilde{\theta}}}:=\left(\left(\frac{\partial L_{\hat{\theta}}}{\partial u}\right)^{\mathrm{T}},\left(\frac{\partial L_{\hat{\theta}}}{\partial \lambda}\right)^{\mathrm{T}}, \epsilon^{\mathrm{T}}, \tilde{\theta}^{\mathrm{T}}\right)^{\mathrm{T}}$ and the estimated Lagrangian function:

$L_{\hat{\theta}}(t, x, u, \lambda, \hat{\theta}):=J(t, x, u)+(k(t, x)-\Phi(t, x, u, \hat{\theta}))^{\mathrm{T}} \lambda$.

We will in what follows prove that the time and statevarying optimal set $\mathcal{O}_{u \lambda \tilde{\theta}}(t, x)$, in a certain sense is
UGS/UGAS with respect to (27) and the certainty equivalent control allocation algorithm:

$$
\begin{aligned}
& \left(\begin{array}{c}
\dot{u} \\
\dot{\lambda}
\end{array}\right)=-\Gamma \mathbb{H}_{\hat{\theta}}\left(\begin{array}{c}
\frac{\partial L_{\hat{\theta}}}{\partial u} \\
\frac{\partial L_{\hat{\theta}}}{\partial \lambda}
\end{array}\right)-u_{f f \hat{\theta}} \\
& \dot{\hat{\theta}}=\Gamma_{\hat{\theta}}^{-1}\left(\Phi_{\theta}(t, x, u)\right)^{\mathrm{T}}\left(g_{x}(t, x)\right)^{\mathrm{T}}\left(\Gamma_{\epsilon} \epsilon+\left(\frac{\partial^{2} L_{\hat{\theta}}}{\partial x \partial u}\right)^{\mathrm{T}} \frac{\partial L_{\hat{\theta}}}{\partial u}\right) \\
& +\Gamma_{\hat{\theta}}^{-1}\left(\Phi_{\theta}(t, x, u)\right)^{\mathrm{T}}\left(g_{x}(t, x)\right)^{\mathrm{T}}\left(\frac{\partial^{2} L_{\hat{\theta}}}{\partial x \partial \lambda}\right)^{\mathrm{T}} \frac{\partial L_{\hat{\theta}}}{\partial \lambda} .
\end{aligned}
$$

where $\mathbb{H}_{\hat{\theta}}:=\left(\begin{array}{cc}\frac{\partial^{2} L_{\hat{\theta}}}{\partial u^{2}} & \frac{\partial^{2} L_{\hat{\theta}}}{\partial \lambda \partial u} \\ \frac{\partial^{2} L_{\hat{\theta}}}{\partial u \partial \lambda} & 0\end{array}\right)$, the matrices $\Gamma, \Gamma_{\epsilon}$ and $\Gamma_{\hat{\theta}}$, are symmetric positive definite and $u_{f f \hat{\theta}}$ is a feedforward like term given by:

$$
\begin{aligned}
u_{f f \hat{\theta}}: & =\mathbb{H}_{\hat{\theta}}^{-1}\left(\left(\begin{array}{c}
\frac{\partial^{2} L}{\partial t \partial u} \\
\frac{\partial^{2} L}{\partial t \partial \lambda}
\end{array}\right)+\left(\begin{array}{c}
\frac{\partial^{2} L}{\partial x \partial u} \\
\frac{\partial^{2} L}{\partial x \partial \lambda}
\end{array}\right) f(t, x)+\left(\begin{array}{c}
\frac{\partial^{2} L_{\hat{\theta}}}{\partial \hat{\theta} \partial u} \\
\frac{\partial^{2} L_{\hat{\theta}}}{\partial \hat{\theta} \partial \lambda}
\end{array}\right) \dot{\hat{\theta}}\right) \\
& +\mathbb{H}_{\hat{\theta}}^{-1}\left(\begin{array}{c}
\frac{\partial^{2} L}{\partial x \partial u} \\
\frac{\partial^{2} L}{\partial x \partial \lambda}
\end{array}\right) g(t, x)(k(t, x)-\Phi(t, x, u, \hat{\theta}))
\end{aligned}
$$

if $\operatorname{det}\left(\frac{\partial^{2} L}{\partial u^{2}}\right)>\epsilon$ or $u_{f f \hat{\theta}}:=0$ if $\operatorname{det}\left(\frac{\partial^{2} L}{\partial u^{2}}\right)<\epsilon$, where $\left(k_{1}\right)^{r}>\epsilon>0 . k_{1}$ is defined in the following assumption which both guarantee existence of the proposed update laws and the time-varying first order optimal set,

$\mathcal{O}_{u \lambda}(t, x):=\left\{\left(u^{\mathrm{T}}, \lambda^{\mathrm{T}}\right)^{\mathrm{T}} \in \mathbb{R}^{r+d} \mid\left(\left(\frac{\partial L}{\partial u}\right)^{\mathrm{T}},\left(\frac{\partial L}{\partial \lambda}\right)^{\mathrm{T}}\right)^{\mathrm{T}}=0\right\}$.

Assumption 3 (Optimal Control Allocation)

a) The cost function $J: \mathbb{R}_{\geq t_{0}} \times \mathbb{R}^{n \times r} \rightarrow \mathbb{R}$ is twice differentiable and $J(t, x, u) \longrightarrow \infty$ as $|u| \rightarrow \infty$. Further there exist continuous increasing functions $\varsigma_{\partial J}, \varsigma_{\partial^{2} J}$ : $\mathbb{R}_{\geq 0} \rightarrow \mathbb{R}_{\geq 0}$ such that $\left|\frac{\partial J}{\partial u}\right| \leq \varsigma_{\partial J}\left(\left|\left(x^{T}, u^{T}\right)\right|\right)$ and $\left|\frac{\partial^{2} J}{\partial t \partial u}\right|+\left|\frac{\partial^{2} J}{\partial x \partial u}\right| \leq \varsigma_{\partial^{2} J}\left(\left|\left(x^{T}, u^{T}\right)\right|\right) \forall t, x$ and $u$.

b) There exists constants $k_{2}>k_{1}>0$, such that $\forall t, x$, $\theta$ and $\left(u^{T}, \lambda^{T}\right)^{T} \notin \mathcal{O}_{u \lambda}^{0}$

$$
k_{1} I<\frac{\partial^{2} L}{\partial u^{2}}(t, x, u, \lambda, \theta) \leq k_{2} I
$$

where $\mathcal{O}_{u \lambda}^{0}$ is the interior of $\mathcal{O}_{u \lambda}$. If $\left(u^{T}, \lambda^{T}\right)^{T} \in \mathcal{O}_{u \lambda}^{0}$ then the lower bound is replaced by $\frac{\partial^{2} L}{\partial u^{2}} \geq 0$.

Remark 4 The second order sufficient conditions in Theorem 12.6 in (Nocedal \& Wright 1999) are satisfied for all $t, x, u, \lambda$ and $\theta$ by Assumption 1 and 3 , thus the set $\mathcal{O}_{u \lambda}(t, x)$ describes global optimal solutions of the problem (3).

Lemma 2 If Assumptions 1, 2 a) and 3 are satisfied, then $\mathcal{O}_{u \lambda}$ is non-empty for all $t, x$ and $\theta$. Further for allt, $x, \theta$ and $(u, \lambda) \in \mathcal{O}_{u \lambda}$, there exist a continuous function $\varsigma_{u \lambda}: \mathbb{R}_{\geq 0} \rightarrow \mathbb{R}_{\geq 0}$ such that:

$$
\left|\left(u^{T}, \lambda^{T}\right)^{T}\right| \leq \varsigma_{\mathcal{O}_{u \lambda}}\left(\left|\left(x^{T}, \theta\right)^{T}\right|\right)
$$


PROOF. See Appendix A.

The idea of proving stability and convergence of the set $\mathcal{O}_{u \lambda \tilde{\theta}}(t, x)$, relies on the construction of the Lyapunovlike function:

$$
\begin{aligned}
V_{u \lambda \tilde{\theta}}(t, x, u, \lambda, \epsilon, \tilde{\theta}): & =\frac{1}{2}\left(\left(\frac{\partial L_{\hat{\theta}}}{\partial u}\right)^{\mathrm{T}} \frac{\partial L_{\hat{\theta}}}{\partial u}+\left(\frac{\partial L_{\hat{\theta}}}{\partial \lambda}\right)^{\mathrm{T}} \frac{\partial L_{\hat{\theta}}}{\partial \lambda}\right) \\
& +\frac{\epsilon^{\mathrm{T}} \Gamma_{\epsilon} \epsilon}{2}+\frac{\tilde{\theta}^{\mathrm{T}} \Gamma_{\hat{\theta}} \tilde{\theta}}{2} .
\end{aligned}
$$

Its time derivative along the trajectories of the considered system is:

$$
\begin{aligned}
& \dot{V}_{u \lambda \hat{\theta}}=\left(\left(\frac{\partial L_{\hat{\theta}}}{\partial u}\right)^{\mathrm{T}} \frac{\partial^{2} L_{\hat{\theta}}}{\partial u^{2}}+\left(\frac{\partial L_{\hat{\theta}}}{\partial \lambda}\right)^{\mathrm{T}} \frac{\partial^{2} L_{\hat{\theta}}}{\partial u \partial \lambda}\right) \dot{u} \\
& +\left(\frac{\partial L_{\hat{\theta}}}{\partial u}\right)^{\mathrm{T}} \frac{\partial^{2} L_{\hat{\theta}}}{\partial \lambda \partial u} \dot{\lambda}+\left(\left(\frac{\partial L_{\hat{\theta}}}{\partial u}\right)^{\mathrm{T}} \frac{\partial^{2} L_{\hat{\theta}}}{\partial x \partial u}+\left(\frac{\partial L_{\hat{\theta}}}{\partial \lambda}\right)^{\mathrm{T}} \frac{\partial^{2} L_{\hat{\theta}}}{\partial x \partial \lambda}\right) \dot{x} \\
& -\left(\left(\frac{\partial L_{\hat{\theta}}}{\partial u}\right)^{\mathrm{T}} \frac{\partial^{2} L_{\hat{\theta}}}{\partial \hat{\theta} \partial u}+\left(\frac{\partial L_{\hat{\theta}}}{\partial \lambda}\right)^{\mathrm{T}} \frac{\partial^{2} L_{\hat{\theta}}}{\partial \hat{\theta} \partial \lambda}\right)^{\tilde{\theta}} \\
& +\left(\frac{\partial L_{\hat{\theta}}}{\partial u}\right)^{\mathrm{T}} \frac{\partial^{2} L_{\hat{\theta}}}{\partial t \partial u}+\left(\frac{\partial L_{\hat{\theta}}}{\partial \lambda}\right)^{\mathrm{T}} \frac{\partial^{2} L_{\hat{\theta}}}{\partial t \partial \lambda}+\epsilon^{\mathrm{T}} \dot{\epsilon}+\tilde{\theta}^{\mathrm{T}} \Gamma_{\hat{\theta}} \dot{\tilde{\theta}} .
\end{aligned}
$$

Further by (27), (30) and (31), we get

$$
\begin{aligned}
\dot{V}_{u \lambda \hat{\theta}}= & -\left(\left(\frac{\partial L_{\hat{\theta}}}{\partial u}\right)^{\mathrm{T}},\left(\frac{\partial L_{\hat{\theta}}}{\partial \lambda}\right)^{\mathrm{T}}\right) \mathbb{H}_{\hat{\theta}} \Gamma \mathbb{H}_{\hat{\theta}}\left(\left(\frac{\partial L_{\hat{\theta}}}{\partial u}\right)^{\mathrm{T}},\left(\frac{\partial L_{\hat{\theta}}}{\partial \lambda}\right)^{\mathrm{T}}\right)^{\mathrm{T}} \\
& -\epsilon^{\mathrm{T}} \Gamma_{\epsilon} A \epsilon \\
\leq & -c_{\hat{\theta}}\left(\left(\frac{\partial L_{\hat{\theta}}}{\partial u}\right)^{\mathrm{T}} \frac{\partial L_{\hat{\theta}}}{\partial u}+\left(\frac{\partial L_{\hat{\theta}}}{\partial \lambda}\right)^{\mathrm{T}} \frac{\partial L_{\hat{\theta}}}{\partial \lambda}\right)-\epsilon^{\mathrm{T}} \Gamma_{\epsilon} A \epsilon
\end{aligned}
$$

where $c_{\hat{\theta}}:=\inf _{t} \lambda_{\min }\left(\mathbb{H}_{\hat{\theta}} \Gamma \mathbb{H}_{\hat{\theta}}\right)>0$. We formalize the result, based on set-stability.

Proposition 1 Let Assumptions 1, 2 a) and 3 be satisfied. Then if $x(t)$ exists for all $t$, the set $\mathcal{O}_{u \lambda \tilde{\theta}}(t, x(t))$ is $U G S$ with respect to the system (27), (30) and (31), and $\left(\epsilon, \frac{\partial L_{\hat{\theta}}}{\partial u}, \frac{\partial L_{\hat{\theta}}}{\partial \lambda}\right)$ converges asymptotically to zero.

PROOF. In order to prove this result we show that i) $\mathcal{O}_{u \lambda \tilde{\theta}}(t, x(t))$ is a closed forward invariant set with respect to system (27), (30) and (31), ii) the system (27), (30) and (31) is finite escape time detectable through $\left|z_{u \lambda \tilde{\theta}}\right|_{\mathcal{O}_{u \lambda \tilde{\theta}}}$, where $z_{u \lambda \tilde{\theta}}:=\left(u^{T}, \lambda^{T}, \epsilon^{T}, \tilde{\theta}^{T}\right)^{T}$, and that iii) $\bar{V}_{u \lambda \tilde{\theta}}\left(t, z_{u \lambda \tilde{\theta}}\right):=V_{u \lambda \tilde{\theta}}(t, x(t), u, \lambda, \epsilon, \tilde{\theta})$, is a radially unbounded Lyapunov function.

i) Define $G(t, x, u, \lambda, \tilde{\theta}):=\left(\epsilon, \tilde{\theta},{\frac{\partial L_{\hat{\theta}}}{\partial u}}^{T},{\frac{\partial L_{\hat{\theta}}}{\partial \lambda}}^{T}\right)^{T}$.

From Proposition 1.1.9 b) in (Bertsekas, Nedic \&
Ozdaglar 2003) we have that $G: \mathbb{R}^{q} \rightarrow \mathbb{R}^{q_{G}}$ is continuous iff $G^{-1}(U)$ is closed in $\mathbb{R}^{q}$ for every closed $U$ in $\mathbb{R}^{q_{G}}$. From the definition of $\mathcal{O}_{u \lambda \tilde{\theta}}, U=\{0\}$, and since $G$ is continuous (by Assumption 1 - 3), $\mathcal{O}_{u \lambda \tilde{\theta}}$ is a closed set. The set is forward invariant if at $t_{1}, G\left(t_{1}, x\left(t_{1}\right), u\left(t_{1}\right), \lambda\left(t_{1}\right), \tilde{\theta}\left(t_{1}\right)\right)=0$ and $\frac{d(G(t, x, u, \lambda, \tilde{\theta}))}{d t}=0 \forall t \geq t_{1}$ with respect to (27), (30), (31) and (28). Since there exist a continuous solution of (30) as long as $x$ exist, we only need to check this condition on the boundary of $\mathcal{O}_{u \lambda \tilde{\theta}}(t, x(t))$ (Note that $\operatorname{det}\left(\mathbb{H}_{\hat{\theta}}\right) \neq 0$ on the boundary of $\mathcal{O}_{u \lambda \tilde{\theta}}(t, x(t))$ by Assumption 1d) and 3b)). We get $G\left(t_{1}, x\left(t_{1}\right), u\left(t_{1}\right), \lambda\left(t_{1}\right), \tilde{\theta}\left(t_{1}\right)\right)=0 \Rightarrow(\dot{\epsilon}, \dot{\hat{\theta}})=0$ and it remains to prove that $\left(\frac{d \partial L_{\hat{\theta}}}{d t \partial \lambda}, \frac{d \partial L_{\hat{\theta}}}{d t \partial u}\right)=0$. $\frac{d \partial L_{\hat{\theta}}}{d t \partial \lambda}=\frac{\partial^{2} L_{\hat{\theta}}}{\partial t \partial \lambda}+\frac{\partial^{2} L_{\hat{\theta}}}{\partial x \partial \lambda}+\frac{\partial^{2} L_{\hat{\theta}}}{\partial u \partial \lambda}+\frac{\partial^{2} L_{\hat{\theta}}}{\partial \hat{\theta}} \dot{\hat{\theta}}$ and $\frac{d \partial L_{\hat{\theta}}}{d t \partial u}=\frac{\partial^{2} L_{\hat{\theta}}}{\partial t \partial u}+\frac{\partial^{2} L_{\hat{\theta}}}{\partial x \partial u} \dot{x}+\frac{\partial^{2} L_{\hat{\theta}}}{\partial u \partial u} \dot{u}+\frac{\partial^{2} L_{\hat{\theta}}}{\partial \lambda \partial u} \dot{\lambda}+\frac{\partial^{2} L_{\hat{\theta}}}{\partial \hat{\theta} \partial u}$, thus

$$
\left(\begin{array}{l}
\frac{d \partial L_{\hat{\theta}}}{d t \partial u} \\
\frac{d \partial L_{\hat{\theta}}}{d t \partial \lambda}
\end{array}\right)=\mathbb{H}_{\hat{\theta}}\left(\begin{array}{l}
\dot{u} \\
\dot{\lambda}
\end{array}\right)+\mathbb{H}_{\hat{\theta}} u_{f f \hat{\theta}}=-\mathbb{H}_{\hat{\theta}} \Gamma \mathbb{H}_{\hat{\theta}}\left(\begin{array}{c}
\frac{\partial L_{\hat{\theta}}}{\partial u} \\
\frac{\partial L_{\hat{\theta}}}{\partial \lambda}
\end{array}\right)=0 .
$$

ii) Since $x(t)$ is assumed to be forward complete, it follows from Lemma 2 that there always exists a pair $\left(u^{* T}, \lambda^{* T}\right)^{T}$ bounded by $x$ and $\hat{\theta}=\theta-\tilde{\theta}$ that satisfy the time-varying first order optimal conditions $\left({\frac{\partial L_{\hat{\theta}}}{\partial u}}^{T}, \frac{\partial L_{\hat{\theta}}}{\partial \lambda}\right)=0$, and thus the system (27), (30) and (31) is finite escape time detectable through $\left|z_{u \lambda \tilde{\theta}}\right|_{\mathcal{O}_{u \lambda \tilde{\theta}}}$.

iii) In the proof of Lemma, change $L$ with $L_{\hat{\theta}}$ such that (A.3) become

$$
\left(\frac{\partial L_{\hat{\theta}}}{\partial u}\right)^{T} \frac{\partial L_{\hat{\theta}}}{\partial u}+\left(\frac{\partial L_{\hat{\theta}}}{\partial \lambda}\right)^{T} \frac{\partial L_{\hat{\theta}}}{\partial \lambda}=\left(\begin{array}{c}
u-u^{*} \\
\lambda-\lambda^{*}
\end{array}\right)^{T} \mathbb{H}_{\hat{\theta} *}^{T} \mathbb{H}_{\hat{\theta} *}\left(\begin{array}{c}
u-u^{*} \\
\lambda-\lambda^{*}
\end{array}\right)
$$

and it follows that there exists constants $\kappa_{2} \geq \kappa_{1}>0$ such that $\bar{\kappa}_{1}\left|z_{u \lambda}\right|_{\mathcal{O}_{u \lambda}}^{2} \leq\left(\left(\frac{\partial L_{\hat{\theta}}}{\partial u}\right)^{T} \frac{\partial L_{\hat{\theta}}}{\partial u}+\left(\frac{\partial L_{\hat{\theta}}}{\partial \lambda}\right)^{T} \frac{\partial L_{\hat{\theta}}}{\partial \lambda}\right) \leq$ $\bar{\kappa}_{2}\left|z_{u \lambda}\right|_{\mathcal{O}_{u \lambda}}^{2}$ since $\mathbb{H}_{\hat{\theta} *}^{T} \mathbb{H}_{\hat{\theta} *}$ is positive definite and bounded from Assumption 1 and 3. By a similar argument, in (A.4) change $P_{* c}$ with $\frac{\partial^{2} L_{\hat{\theta}}}{\partial u^{2}}, \mathbb{H}_{\hat{\theta}}$ is non-singular, hence the control allocation law (30) always exist and, $\kappa_{1}\left|z_{u \lambda \tilde{\theta}}\right|_{\mathcal{O}_{u \lambda \tilde{\theta}}} \leq \bar{V}_{u \lambda \tilde{\theta}}\left(t, z_{u \lambda \tilde{\theta}}\right) \leq$ $\kappa_{2}\left|z_{u \lambda \tilde{\theta}}\right|_{\mathcal{O}_{u \lambda \tilde{\theta}}}$, where $\kappa_{1}:=\frac{1}{2} \min \left(\bar{\kappa}_{1}, \Gamma_{\epsilon}, \Gamma_{\hat{\theta}}\right) \kappa_{2}:=$ $\frac{1}{2} \max \left(\bar{\kappa}_{2}, \Gamma_{\epsilon}, \Gamma_{\hat{\theta}}\right)$, such that $\bar{V}_{u \lambda \tilde{\theta}}\left(t, z_{u \lambda \tilde{\theta}}\right)$ is a radially unbounded Lyapunov function.

Since (34) is negative semidefinite, UGS of the set $\mathcal{O}_{u \lambda \tilde{\theta}}$ can be concluded by Theorem 1. The convergence result follows by Barbalat's lemma

Corollary 2 Let Assumptions 1, 2 a) and 3 be satisfied and $\left|z_{u \lambda \tilde{\theta} 0}\right|_{\mathcal{O}_{u \lambda \tilde{\theta}}}<r$ where $r>0$. If $x(t)$ exists 
for $t \in\left[t_{0}, T\right)$ where $T \geq t_{0}$, then there exists a positive constant $B(r)>0$ such that for all $t \in\left[t_{0}, T\right)$, $\left|z_{u \lambda \tilde{\theta}}(t)\right|_{\mathcal{O}_{u \lambda \tilde{\theta}}(t, x(t))} \leq B(r)$.

PROOF. Since $\dot{\bar{V}}_{u \lambda \tilde{\theta}} \leq 0$ and $\bar{V}_{u \lambda \tilde{\theta}}\left(t, z_{u \lambda \tilde{\theta}}(t)\right) \leq$ $\bar{V}_{u \lambda \tilde{\theta}}\left(t_{0}, z_{u \lambda \tilde{\theta}}\left(t_{0}\right)\right)$ for all $t \in\left[t_{0}, T\right)$. From iii) in the proof of Proposition $1 \kappa_{1}\left|z_{u \lambda \tilde{\theta}}\right|_{\mathcal{O}_{u \lambda \tilde{\theta}}} \leq \bar{V}_{u \lambda \tilde{\theta}}\left(t, z_{u \lambda \tilde{\theta}}\right) \leq$ $\kappa_{2}\left|z_{u \lambda \tilde{\theta}}\right|_{\mathcal{O}_{u \lambda \tilde{\theta}}}$ for all $t \in\left[t_{0}, T\right)$, thus it follows that $\left|z_{u \lambda \tilde{\theta}}(t)\right|_{\mathcal{O}_{u \lambda \tilde{\theta}}(t, x(t))} \leq \sqrt{\frac{1}{\kappa_{1}} \bar{V}_{u \lambda \tilde{\theta}}\left(t, z_{u \lambda \tilde{\theta}}(t)\right)} \leq$ $\sqrt{\frac{1}{\kappa_{1}} \bar{V}_{u \lambda \tilde{\theta}}\left(t_{0}, z_{u \lambda \tilde{\theta}}\left(t_{0}\right)\right)} \leq \sqrt{\frac{\kappa_{2}}{\kappa_{1}}}\left|z_{u \lambda \tilde{\theta} 0}\right|_{\mathcal{O}_{u \lambda \tilde{\theta}}}=: B(r)$

Remark 5 Provided that the gain matrix, $\Gamma>0$, is bounded away from zero, $\Gamma$ may be chosen time-varying. If for example $\Gamma=\gamma\left(\mathbb{H}_{\hat{\theta}} \mathbb{H}_{\hat{\theta}}\right)^{-1}$ for some $\gamma>0$, then

$$
\left(\begin{array}{c}
\dot{u} \\
\dot{\lambda}
\end{array}\right)=-\gamma \mathbb{H}_{\hat{\theta}}^{-1}\left(\begin{array}{c}
\frac{\partial L_{\hat{\theta}}}{\partial u} \\
\frac{\partial L_{\hat{\theta}}}{\partial \lambda}
\end{array}\right)-\mathbb{H}_{\hat{\theta}}^{-1} u_{f f}
$$

where the first term is the Newton direction when $L_{\hat{\theta}}$ is considered the cost function to be minimized. In case $\mathbb{H}_{\hat{\theta}} \mathbb{H}_{\hat{\theta}}$ is poorly conditioned one may choose $\Gamma=\gamma\left(\mathbb{H}_{\hat{\theta}} \mathbb{H}_{\hat{\theta}}+\rho I\right)^{-1}$ for some $\rho>0$, to avoid $c_{\hat{\theta}}$ in (34) from being small.

Assumption 4 (Persistence of Excitation) The signal matrix $\Phi_{g}(t):=g_{x}(t, x(t)) \Phi_{\theta}(t, x(t), u(t))$ is Persistently Excited (PE), which means that there exist constants $T$ and $\gamma>0$, such that

$$
\int_{t}^{t+T} \Phi_{g}(\tau)^{T} \Phi_{g}(\tau) d \tau \geq \gamma I, \forall t>t_{0}
$$

Remark 6 The system trajectories $x(t)$ defined by (1) typically represents the tracking error i.e. $x:=x_{s}-x_{d}$, where $x_{s}$ is states vector of the system, and $x_{d}$ represents the desired reference for these states. This means that $P E$ assumption on $\Phi_{g}(t)$ is dependent on the reference trajectory $x_{d}$, in addition to disturbances and noise, and imply that some "richness" properties of these signals are satisfied.

Proposition 2 Let $x(t)$ be $U G B$, then if Assumption 4 and the assumptions of Proposition 1 are satisfied, the set $\mathcal{O}_{\text {u } \tilde{\theta}}$ is UGAS with respect to system (26), (27), (30) and (31).

\section{PROOF. See Appendix B}

Unless the PE condition is satisfied for $\Phi_{g}$, only stability of the optimal set is guaranteed. Thus in the sense of achieving asymptotic optimality, parameter convergence is of importance.

\subsubsection{Stability of the combined control and certainty} equivalent control allocation

The optimal set for the combined control and certainty equivalent control allocation problem is define by:

$$
\mathcal{O}_{x u \lambda \tilde{\theta}}(t):=\mathcal{O}_{x}(t) \times \mathcal{O}_{u \lambda \tilde{\theta}}(t, 0) .
$$

In the framework of cascaded systems, we consider (30), (31) together with $(27)$ to be the perturbing system $\left(\Sigma_{2}\right)$, while $(28)$ represents the perturbed system $\left(\Sigma_{1}\right)$. Loosely explained, we will use Lemma 1 to conclude UGAS of the set $\mathcal{O}_{x u \lambda \tilde{\theta}}$ if, $\mathcal{O}_{x}$ and $\mathcal{O}_{u \lambda \tilde{\theta}}$ individually are UGAS (which we already have established) and the combined system is UGB with respect to $\mathcal{O}_{x u \lambda \tilde{\theta}}$.

Before establishing the main results of this section, we need to state an assumption on the interconnection term between the two systems. We start by stating the following property:

Lemma 3 By Assumption 1 and Lemma 2 there exists continuous functions $\varsigma_{x}, \varsigma_{x u}, \varsigma_{u}: \mathbb{R}_{\geq 0} \rightarrow \mathbb{R}_{\geq 0}$, such that

$$
\left|\Phi_{\theta}(t, x, u)\right| \leq \varsigma_{x}(|x|) \varsigma_{x u}(|x|)+\varsigma_{x}(|x|) \varsigma_{u}\left(\left|z_{u \lambda \tilde{\theta}}\right|_{\mathcal{O}_{u \lambda \tilde{\theta}}}\right) .
$$

PROOF. This can bee seen by applying (Mazenc \& Praly 1996)'s lemma B.1

\section{Assumption 2 (Continued)}

c) The function, $\alpha_{k}$, from Assumption 2 b) has the following additional property:

$$
\alpha_{k}^{-1}(|x|) \alpha_{x 3}(|x|) \geq \alpha_{x 4}(|x|) \varsigma_{x \max }(|x|),
$$

$$
\text { where } \varsigma_{x \max }(|x|):=\max \left(1, \varsigma_{x}(|x|), \varsigma_{x}(|x|) \varsigma_{x u}(|x|)\right) \text {. }
$$

Remark 7 If there exists a constant $K>0$ such that $\varsigma_{x \max }(|x|) \leq K \forall x$, which is common in a mechanical system, Assumption 2 c) is satisfied.

Next we consider the closed loop of the plant, the virtual controller and the adaptive dynamic control allocation law:

Proposition 3 If Assumptions 1-3 are satisfied, then the set $\mathcal{O}_{x u \lambda \tilde{\theta}}$ is UGS, with respect to system (28),(30), (31) and (27). If in addition Assumption 4 is satisfied, then $\mathcal{O}_{x u \lambda \tilde{\theta}}$ is UGAS with respect to (28),(30), (31) and (27).

$\boldsymbol{P R O O F}$. The main part of this proof is to prove boundedness, completeness and invoke Lemma 1. Let $\left|z_{x u \lambda \tilde{\theta} 0}\right|_{\mathcal{O}_{x u \lambda \tilde{\theta}}} \leq r$, where $r>0$, and assume that $\left|z_{x}(t)\right|_{\mathcal{O}_{x}}$ escapes to infinity at $T$. Then for any constant $M(r)$ there exists a $t \in\left[t_{0}, T\right)$ such that $M(r) \leq\left|z_{x}(t)\right|_{\mathcal{O}_{x}}$. In what follows we show that $M(r)$ can not be chosen arbitrarily. Define $v\left(t, z_{x}\right):=V_{x}(t, x)$ such that 


$$
\begin{aligned}
& \dot{v} \leq-\alpha_{x 3}\left(\left|z_{x}\right|_{\mathcal{O}_{x}}\right)+\frac{\partial V_{x}}{\partial x} g(t, x)(k(t, x)-\Phi(t, x, u, \hat{\theta})) \\
& +\frac{\partial V_{x}}{\partial x} g_{x}(t, x) \Phi_{\theta}(t, x, u) \tilde{\theta} \\
& \leq-\alpha_{x 3}\left(\left|z_{x}\right|_{\mathcal{O}_{x}}\right)+\left|\frac{\partial V_{x}}{\partial x}\right||g(t, x)|\left|\frac{\partial L_{\hat{\theta}}}{\partial \lambda}(t, x, u, \hat{\theta})\right| \\
& +\left|\frac{\partial V_{x}}{\partial x}\right|\left|g_{x}(t, x)\right|\left|\Phi_{\theta}(t, x, u) \tilde{\theta}\right| \\
& \leq-\alpha_{x 3}\left(\left|z_{x}\right|_{\mathcal{O}_{x}}\right) \\
& +\alpha_{x 4}\left(\left|z_{x}\right|_{\mathcal{O}_{x}}\right) K\left(\kappa_{2}+\left|\Phi_{\theta}(t, x, u)\right|\right)\left|z_{u \lambda \tilde{\theta}}\right|_{\mathcal{O}_{u \lambda \tilde{\theta}}} \cdot
\end{aligned}
$$

From Corollary 2, there exists a positive constant $B(r) \geq$ 0 , for all $t \in\left[t_{0}, T\right)$, such that for $\left|z_{u \lambda \tilde{\theta} 0}\right|_{\mathcal{O}_{u \lambda \tilde{\theta}}} \leq r$, $\left|z_{u \lambda \tilde{\theta}}(t)\right|_{\mathcal{O}_{u \lambda \tilde{\theta}}} \leq B\left(\left|z_{u \lambda \tilde{\theta} 0}\right|_{\mathcal{O}_{u \lambda \tilde{\theta}}}\right) \leq B(r)$. From Assumption 1 and 2 ,

$$
\begin{aligned}
\dot{v} \leq & -\alpha_{x 3}\left(\left|z_{x}\right|_{\mathcal{O}_{x}}\right) \\
& +\alpha_{x 4}\left(\left|z_{x}\right|_{\mathcal{O}_{x}}\right) K\left(\kappa_{2}+\left|\Phi_{\theta}(t, x, u)\right|\right) B(r) \\
\leq & -\alpha_{k}\left(\left|z_{x}\right|_{\mathcal{O}_{x}}\right) \alpha_{x 4}\left(\left|z_{x}\right|_{\mathcal{O}_{x}}\right) \varsigma_{x \max }\left(\left(\left|z_{x}\right|_{\mathcal{O}_{x}}\right)\right. \\
& +\alpha_{x 4}\left(\left|z_{x}\right|_{\mathcal{O}_{x}}\right) K\left(\kappa_{2}+\left|\Phi_{\theta}(t, x, u)\right|\right) B(r) \\
\leq & -\alpha_{x 4}\left(\left|z_{x}\right|_{\mathcal{O}_{x}}\right) \alpha_{k}\left(\left|z_{x}\right|_{\mathcal{O}_{x}}\right) \varsigma_{x \max }\left(\left|z_{x}\right|_{\mathcal{O}_{x}}\right) \\
& +\alpha_{x 4}\left(\left|z_{x}\right|_{\mathcal{O}_{x}}\right) K \kappa_{2} B(r) \\
& +\alpha_{x 4}\left(\left|z_{x}\right|_{\mathcal{O}_{x}}\right) K \varsigma_{x}\left(\left|z_{x}\right|_{\mathcal{O}_{x}}\right) \varsigma_{x u}\left(\left|z_{x}\right|_{\mathcal{O}_{x}}\right) B(r) \\
& +\alpha_{x 4}\left(\left|z_{x}\right|_{\mathcal{O}_{x}}\right) K \varsigma_{x}\left(\left|z_{x}\right|_{\mathcal{O}_{x}}\right) \varsigma_{u}(B(r)) B(r) \\
\leq & -\alpha_{x 4}\left(\left|z_{x}\right|_{\mathcal{O}_{x}}\right) \alpha_{k}\left(\alpha_{x 2}^{-1}(v)\right) \varsigma_{x \max }\left(\left|z_{x}\right|_{\mathcal{O}_{x}}\right) \\
& +\alpha_{x 4}\left(\left|z_{x}\right|_{\mathcal{O}_{x}}\right) K \kappa_{2} B(r) \\
& +\alpha_{x 4}\left(\left|z_{x}\right|_{\mathcal{O}_{x}}\right) K \varsigma_{x}\left(\left|z_{x}\right|_{\mathcal{O}_{x}}\right) \varsigma_{x u}\left(\left|z_{x}\right|_{\mathcal{O}_{x}}\right) B(r) \\
& +\alpha_{x 4}\left(\left|z_{x}\right|_{\mathcal{O}_{x}}\right) K \varsigma_{x}\left(\left|z_{x}\right|_{\mathcal{O}_{x}}\right) \varsigma_{u}(B(r)) B(r) .
\end{aligned}
$$

Thus, if

$$
\left|z_{x 0}\right|_{\mathcal{O}_{x}}>\alpha_{k}^{-1}\left(K\left(\kappa_{2}+1+\varsigma_{u}(B(r))\right) B(r)\right)
$$

then from (39),

$v\left(t_{0}, z_{x 0}\right) \geq v\left(t, z_{x}(t)\right)$ and $\left|z_{x}(t)\right|_{\mathcal{O}_{x}} \leq \alpha_{x 1}^{-1}\left(v\left(z_{x 0}\right)\right) \leq$ $\alpha_{x 1}^{-1}\left(\alpha_{x 2}(r)\right)$, else,

$$
\left|z_{x 0}\right|_{\mathcal{O}_{x}} \leq \alpha_{k}^{-1}\left(K\left(\kappa_{2}+1+\varsigma_{u}(B(r))\right) B(r)\right)
$$

and from (40),

$v\left(t, z_{x}(t)\right) \leq \alpha_{x 2}\left(\alpha_{k}^{-1}\left(K\left(\kappa_{2}+1+\varsigma_{u}(B(r))\right) B(r)\right)\right)$ such that

$\left|z_{x}(t)\right|_{\mathcal{O}_{x}} \leq \alpha_{x 1}^{-1}\left(\alpha_{x 2}\left(\alpha_{k}^{-1}\left(K\left(\kappa_{2}+1+\varsigma_{u}(B(r))\right) B(r)\right)\right)\right)$. By choosing

$M(r):=\max \left(\alpha_{x 1}^{-1}\left(\alpha_{x 2}(r)\right)\right.$,

$\left.\alpha_{x 1}^{-1}\left(\alpha_{x 2}\left(\alpha_{k}^{-1}\left(K\left(\kappa_{2}+1+\varsigma_{u}(B(r))\right) B(r)\right)\right)\right)\right)$,

the assumption of $\left|z_{x}(t)\right|_{\mathcal{O}_{x}}$ escaping to infinity is contradicted, since $M(r)>\left|z_{x}(t)\right|_{\mathcal{O}_{x}}$ and $|\cdot|_{\mathcal{O}}$ is finite escape time detectable. Further more $\mathcal{O}_{x}$ is UGB. From Propositions 1 and 2 and the assumptions of these propositions, the assumptions of Lemma 1 and Corollary 1 are satisfied and the result is proved
Proposition 3 implies that the time-varying optimal set $\mathcal{O}_{x u \lambda \tilde{\theta}}(t)$ is uniformly stable, and in addition uniformly attractive if Assumption 4 is satisfied. Thus optimal control allocation is achieved asymptotically for the closed loop.

A local version of this result can be proven using Corollary 1.

Corollary 3 If for $u \in \mathbb{U} \subset \mathbb{R}^{r}$ there exist constant $c_{x}>$ 0 such that for $|x| \leq c_{x}$ the domain $\mathbb{U}_{z} \subset \mathbb{R}_{\geq 0} \times \mathbb{R}^{n} \times \mathbb{U} \times$ $\mathbb{R}^{d+n+m}$ contain $\mathcal{O}_{x u \lambda \tilde{\theta}}$, then if the Assumptions 1-3 are satisfied, the set $\mathcal{O}_{x u \lambda \tilde{\theta}}$ is US with respect to the system (28), (30), (31) and (27). If in addition Assumption 4 is satisfied, $\mathcal{O}_{x u \lambda \tilde{\theta}}$ is UAS with respect to the system (28), (30), (31) and (27).

PROOF. Since $\mathcal{O}_{x u \lambda \tilde{\theta}} \subset \mathbb{U}_{z}$, there exist a positive constants $r \geq\left|z_{x u \lambda \tilde{\theta} 0}\right|_{\mathcal{O}_{x u \lambda \tilde{\theta}}}$ such that $\left|z_{x}(t)\right|_{\mathcal{O}_{x}} \leq c_{x 0}$ where $0<c_{x 0}<c_{x}$, hence the domain $\mathbb{U}_{u \lambda \tilde{\theta}} \subset \mathbb{R}_{\geq 0} \times \mathbb{U} \times$ $\mathbb{R}^{d+n+m}$ contain $\mathcal{O}_{u \lambda \tilde{\theta}}$. US/UAS of $\mathcal{O}_{u \lambda \tilde{\theta}}$ follows from the Lyapunov-like function $V_{u \lambda \tilde{\theta}}$ and the PE Assumption. UB follows from $\left|z_{x u \lambda \tilde{\theta} 0}\right|_{\mathcal{O}_{x u \lambda}} \leq r$, and the US/UAS property of $\mathcal{O}_{x u \lambda \tilde{\theta}}$ follows from Corollary

Corollary 4 If $\theta$ is known and the Assumptions 1-3 are satisfied then the set $\mathcal{O}_{x u \lambda}:=\mathcal{O}_{x}(t) \times \mathcal{O}_{u \lambda \tilde{\theta}}(t, 0)$. is UGAS with respect to the system (19) and (30).

PROOF. This result follows by working through the above arguments, neglecting the adaptive dynamic and considering a Lyapunov like function of the form $V_{u \lambda}(t, x, u, \lambda):=\frac{1}{2}\left(\left(\frac{\partial L_{\hat{\theta}}}{\partial u}\right)^{T} \frac{\partial L_{\hat{\theta}}}{\partial u}+\left(\frac{\partial L_{\hat{\theta}}}{\partial \lambda}\right)^{T} \frac{\partial L_{\hat{\theta}}}{\partial \lambda}\right)$

Corollary 5 If for $u \in \mathbb{U} \subset \mathbb{R}^{r}$ there exist constant $c_{x}>0$ such that for $|x| \leq c_{x}$ the domain $\mathbb{U}_{z} \subset \mathbb{R}_{\geq 0} \times$ $\mathbb{R}^{n} \times \mathbb{U} \times \mathbb{R}^{d}$ contain $\mathcal{O}_{x u \lambda}$, then if the Assumptions 1 3 are satisfied, the set $\mathcal{O}_{x u \lambda}$ is UAS with respect to the system (19) and (30).

Since the set with respect to system $\Sigma_{2}$ may only be US, due to actuator/effector constraints and parameter uncertainty, only US of the cascade, may be concluded. But if the PE property is satisfied on $\Phi_{g}$, a UAS result may be achieved with both optimal and adaptive convergence.

\section{Example}

In this section simulation results of an over-actuated scaled-model ship, manoeuvred at low-speed, is presented. A 3DOF horizontal plane model described by:

$$
\begin{aligned}
\dot{\eta}_{e} & =R(\psi) \nu \\
\dot{\nu} & =-M^{-1} D \nu+M^{-1}(\tau+b) \\
\tau & =\Phi_{\theta}(u) \theta
\end{aligned}
$$

is considered, where $\eta_{e}:=\left(x_{e}, y_{e}, \psi_{e}\right)^{\mathrm{T}}:=\left(x_{p}-x_{d}, y_{p}-\right.$ $\left.y_{d}, \psi_{p}-\psi_{d}\right)^{\mathrm{T}}$ is the north and east positions and compass heading deviation. Subscript $p$ and $d$ denotes the 
actual and desired states. $\nu:=(u, v, r)^{\mathrm{T}}$ is the bodyfixed velocities, surge, sway and yaw, $\tau$ is the generalized force vector, $b:=\left(b_{1}, b_{2}, b_{3}\right)^{\mathrm{T}}$ is a disturbance due to wind and current and $R(\psi)$ is the rotation matrix function between the body fixed and the earth fixed coordinate frame. The example we present here is based on (Lindegaard \& Fossen 2003), and is also studied in (Johansen 2004) and (Tjønnås \& Johansen 2005). In the considered model there are five force producing devices; the two main propellers aft of the hull, in conjunction with two rudders, and one tunnel thruster going through the hull of the vessel. $\omega_{i}$ denotes the propeller angular velocity and $\delta_{i}$ denotes the rudder deflection. $i=1,2$ denotes the aft actuators, while $i=3$ denotes the tunnel thruster. (41) can be rewritten in the form of (1) and (2) by:

$$
\begin{aligned}
x & :=\left(\xi, \eta_{e}, \nu\right)^{\mathrm{T}}, \quad \theta:=\left(\theta_{1}, \theta_{2}\right)^{\mathrm{T}}, \\
\tau & :=\left(\tau_{1}, \tau_{2}, \tau_{3}\right)^{\mathrm{T}}, \quad u:=\left(\omega_{1}, \omega_{2}, \omega_{3}, \delta_{1}, \delta_{2}\right)^{\mathrm{T}}, \\
\Phi_{\theta}(u) & :=\left(\begin{array}{cc}
X_{1}+X_{2} & 0 \\
Y_{1}+Y_{2} & T_{3} \\
\Phi_{\theta 13} & l_{3, x} T_{3}
\end{array}\right), \\
\Phi_{\theta 13} & :=-l_{1, y} X_{1}+l_{1, x} Y_{1}-l_{2, y} X_{2}+l_{2, x} Y_{2}, \\
X_{i} & :=T_{i}-D_{i}, \quad Y_{i}:=L_{i},
\end{aligned}
$$

where the thruster forces are defined by:

$$
\begin{aligned}
T_{i} & :=\left\{\begin{array}{ll}
k_{T p_{i}} \omega_{i}^{2} & \omega_{i} \geq 0 \\
k_{T n_{i}}\left|\omega_{i}\right| \omega_{i} \omega_{i}<0
\end{array},\right. \\
L_{i} & :=\left\{\begin{array}{ll}
T_{i}\left(1+k_{L n_{i}} \omega_{i}\right)\left(k_{L \delta 1_{i}}+k_{L \delta 2_{i}}\left|\delta_{i}\right|\right) \delta_{i}, & , \omega_{i} \geq 0 \\
0 & , \omega_{i}<0
\end{array},\right. \\
D_{i} & :=\left\{\begin{array}{ll}
T_{i}\left(1+k_{D n_{i}} \omega_{i}\right)\left(k_{D \delta 1_{i}}\left|\delta_{i}\right|+k_{D \delta 2_{i}} \delta_{i}^{2}\right), & , \omega_{i} \geq 0 \\
0 & , \omega_{i}<0
\end{array} .\right.
\end{aligned}
$$

The unknown parameter vector $\theta$ represents thrust losses. $\theta_{2}$ is also related to the parameters $k_{T p_{3}}$ and $k_{T n_{3}}$ in a multiplicative way. This suggest that the estimate of $\theta_{2}$ gives a direct estimate of the tunnel thruster loss factor. The virtual controller

$$
\tau_{c}:=-K_{i} R^{T}(\psi) \xi-K_{p} R^{T}(\psi) \eta_{e}-K_{d} \nu,
$$

proposed in (Lindegaard \& Fossen 2003), stabilizes the system (41) augmented with: $\dot{\zeta}=-\eta_{e}$, where $\zeta:=b-$ $\xi$ and $\dot{\xi}=\eta_{e}$, uniformly and exponentially, for some physically limited yaw rate. The cost function used is:

$$
\begin{aligned}
J(u) & :=\sum_{i=1}^{3} k_{i}\left|\omega_{i}\right| \omega_{i}^{2}+k_{i 2} \omega_{i}^{2}+\sum_{i=1}^{2} q_{i} \delta_{i}^{2}, \\
\left|\omega_{i}\right| & \leq 18 H z, \quad\left|\delta_{i}\right| \leq 35 \mathrm{deg}, \\
k_{1} & =k_{2}=0.01, \quad k_{3}=0.02, \quad q_{1}=q_{2}=500, \\
k_{i 2} & =10^{-3} .
\end{aligned}
$$

The gain matrices are chosen as follows: $K_{p}:=$ $M \cdot \operatorname{diag}(3.13,3.13,12.5) 10^{-2}$,

$K d:=M \cdot \operatorname{diag}(3.75,3.75,7.5) 10^{-1}, K_{I}:=M$ $\operatorname{diag}(0.2,0.2,4) 10^{-3}, A_{\epsilon}:=I_{9 \times 9}, Q_{\theta}:=\operatorname{diag}(1,1)$, $Q_{\epsilon}:=\operatorname{diag}\left(a, 150 \cdot(100,100,1)^{\mathrm{T}}\right), a:=(1,1,1,1,1,1)^{\mathrm{T}}$ and $\Gamma:=\left(\mathbb{H}_{\hat{\theta}}^{\mathrm{T}} W \mathbb{H}_{\hat{\theta}}+\varepsilon I\right)^{-1}$ where

$W:=\operatorname{diag}(1,1,1,1,1,0.6,0.6,0.6)$ and $\varepsilon:=10^{-9}$. The weighting matrix $W$ is chosen such that the deviation of $\left|\frac{\partial L_{\hat{\theta}}}{\partial \lambda}\right|=|k(t, x)-\Phi(t, x, u, \hat{\theta})|$ from zero, is penalized more then the deviation of $\left|\frac{\partial L_{\hat{\theta}}}{\partial u}\right|$ from zero, in the search direction. In order to keep $\hat{\theta}$ from being zero, due to a physical consideration, a projection algorithm can be used. i.e. from (30)

$$
\dot{\hat{\theta}}=\left\{\begin{array}{l}
\Gamma_{\hat{\theta}}^{-1} \xi \\
\Gamma_{\hat{\theta}}^{-1} \xi+\Gamma_{\hat{\theta}}^{-1} \frac{(1,1)^{\mathrm{T}}(1,1)}{(1,1) \Gamma_{\hat{\theta}}^{-1}(1,1)^{\mathrm{T}}} \Gamma_{\hat{\theta}}^{-1} \xi
\end{array}\right.
$$

where (i) is used if $\hat{\theta} \in S^{0}$ or $\theta \in \delta(S)$ and $\left(\Gamma_{\hat{\theta}}^{-1} \xi\right)^{\mathrm{T}}(1,1)^{\mathrm{T}} \leq 0$, and (ii) is used otherwise. Further

$$
\xi:=\Phi_{\theta}(u)^{\mathrm{T}} g_{x}^{\mathrm{T}}\left(\Gamma_{\epsilon} \epsilon+\frac{\partial^{2} L_{\hat{\theta}}^{\mathrm{T}}}{\partial x \partial u} \frac{\partial L_{\hat{\theta}}}{\partial u}+\frac{\partial^{2} L_{\hat{\theta}}^{\mathrm{T}}}{\partial x \partial \lambda} \frac{\partial L_{\hat{\theta}}}{\partial \lambda}\right)
$$

$S:=\left\{\hat{\theta} \in \mathbb{R}^{2} \mid-\min _{i}\left\{\hat{\theta}_{i}\right\} \leq \hat{\theta}_{\min }\right\}, S^{0}$ is the interior of $S$ and $\delta(S)$ is the boundary of $S$ and $\hat{\theta}_{\min }>0$.

Remark 8 In order to satisfy Assumption 1 we replace $\tau$ in (41) by $\tau=\Phi(u, \theta)=\Phi_{\theta}(u) \theta+\Phi_{0}(u)$, where $\Phi_{0}(u):=\varsigma\left(\omega_{1}, \omega_{2}, \omega_{3}\right)^{T}$ and $\varsigma>0$, in the control allocation algorithm. Since $\varsigma$ may be chosen arbitrarily small, this actuator/effector mapping is practically similar to (41), but ensures that the allocation algorithm is well conditioned. Assumption 3 is satisfied locally since for bounded $u$ and $\lambda, k_{i 2}$ ensures that $\frac{\partial^{2} L}{\partial u^{2}}>0$. It is also worth noticing that the optimal control allocation solution in this example, at any time, is a single point.

Based on the wind and current disturbance vector $b:=$ $0.05(1,1,1)^{\mathrm{T}}$ and the parameter vector $\theta:=(0.8,0.9)^{\mathrm{T}}$, the simulation results are presented in the Figures 3-7. Due to the disturbance and reference change, the transients excite the parameter update-law at $t \approx 0$ as well as at $t \approx 200$ and $t \approx 400$, and the estimated parameters converges to the true values. The control objective is satisfied and the commanded virtual controls are tracked closely, by the forces generated by the adaptive control allocation law, except for $t \approx 0, t \approx 200$ and $t \approx 400$, where the control allocation is suboptimal due to initial conditions and actuator saturation: see Figure 7. The simulations are carried out in the MATLAB environment with a sampling rate of $10 \mathrm{~Hz}$. 

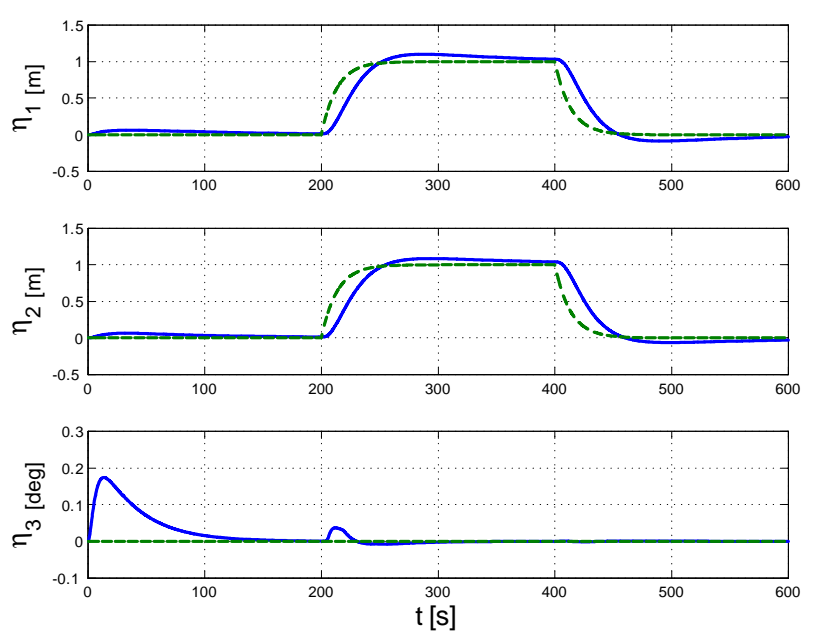

Fig. 3. Simulation results - the solid lines represent positions while the dashed lines represent references
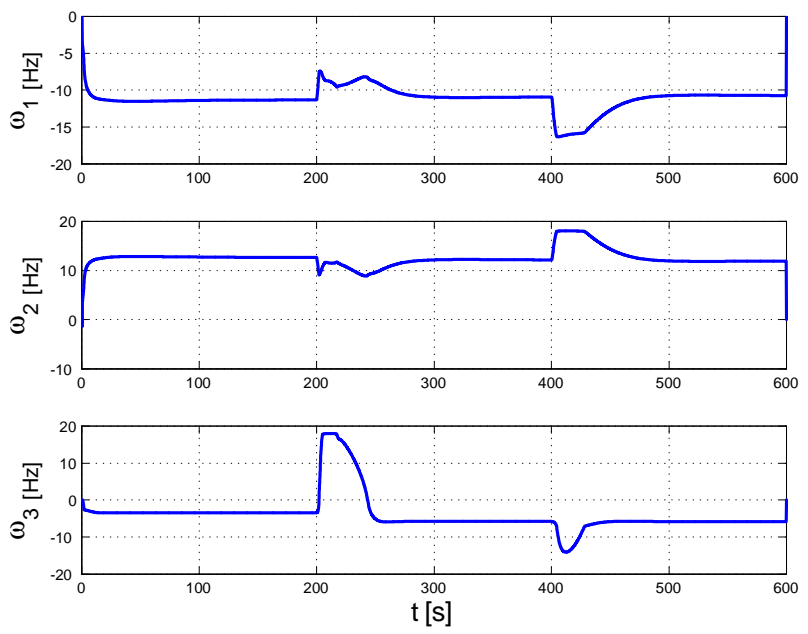

Fig. 4. Simulation results - computed propeller angular velocities by the allocation algorithm

\section{Conclusion}

Based on a control-Lyapunov design approach, an adaptive optimizing nonlinear control allocation algorithm is derived. Under certain assumptions on the system (actuator/effector model) and the control design (growth rate conditions on the Lyapunov function), a cascade result is used to prove, closed-loop, stability and attractivity of a set representing the optimal actuator configuration. Typical applications for the control allocation algorithm are over-actuated mechanical systems, especially systems that exhibits fast dynamics since the algorithm is computational efficient. An automotive example is presented in (Tjønnås \& Johansen 2006).
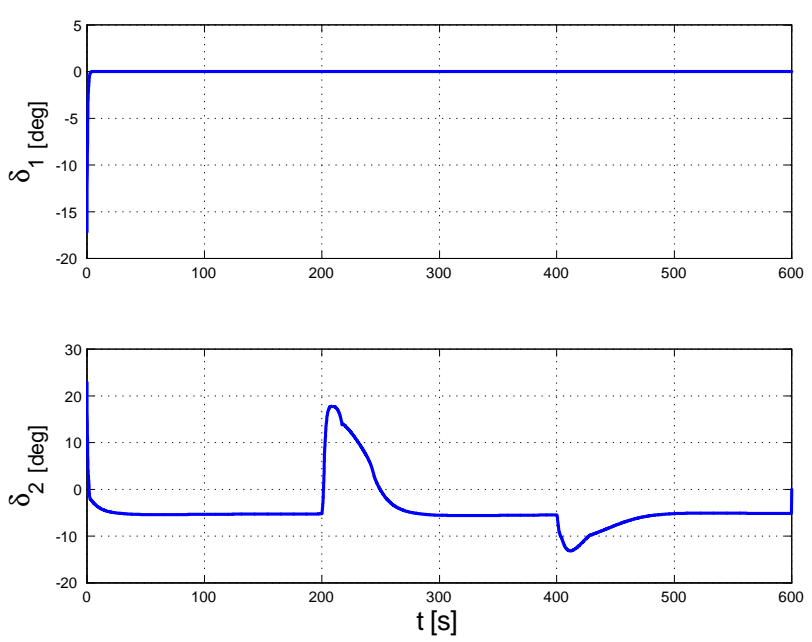

Fig. 5. Simulation results - computed rudder deflection by the allocation algorithm
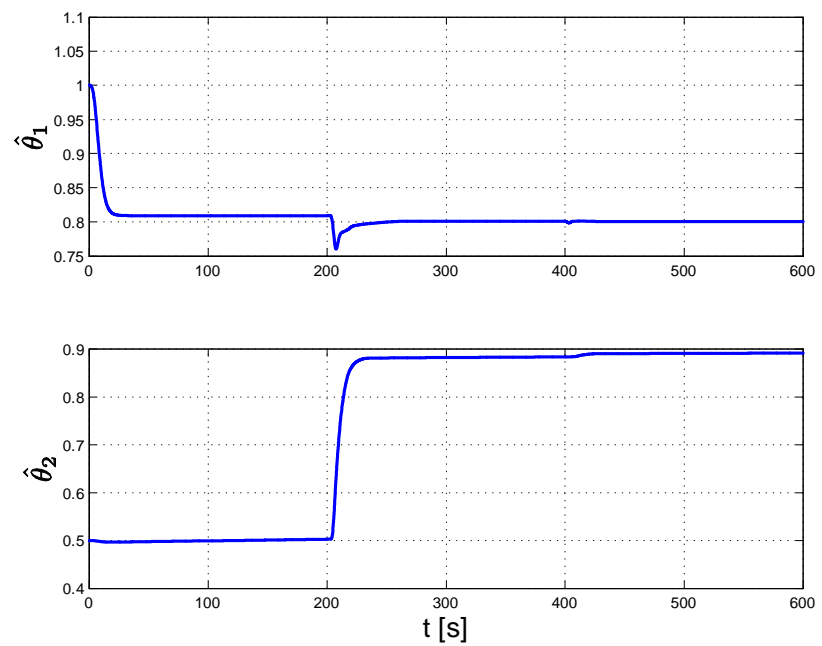

Fig. 6. Simulation results - the parameter adaption

\section{Acknowledgements}

The authors are grateful for insightful comments from Antoine Chaillet, Elena Panteley and Alexey Pavlov. The work is sponsored by the Research Council of Norway through the Strategic University Programme on Computational Methods in Nonlinear Motion Control.

\section{References}

Abrahamson, H., Marsden, J. E. \& Ratiu, T. (1988), Manifolds, Tensor Analysis and applications, 2'nd edn, Springer-Verlag.

Bertsekas, D. P., Nedic, A. \& Ozdaglar, A. E. (2003), Convex Analysis and Optimization, Athena Scientific.

Bodson, M. (2002), 'Evaluation of optimization methods for control allocation', J. Guidance, Control and Dynamics 25, 703-711.

Buffington, J. M., Enns, D. F. \& Teel, A. R. (1998), 'Control allocation and zero dynamics', J. Guidance, Control and Dynamics 21, 458-464. 

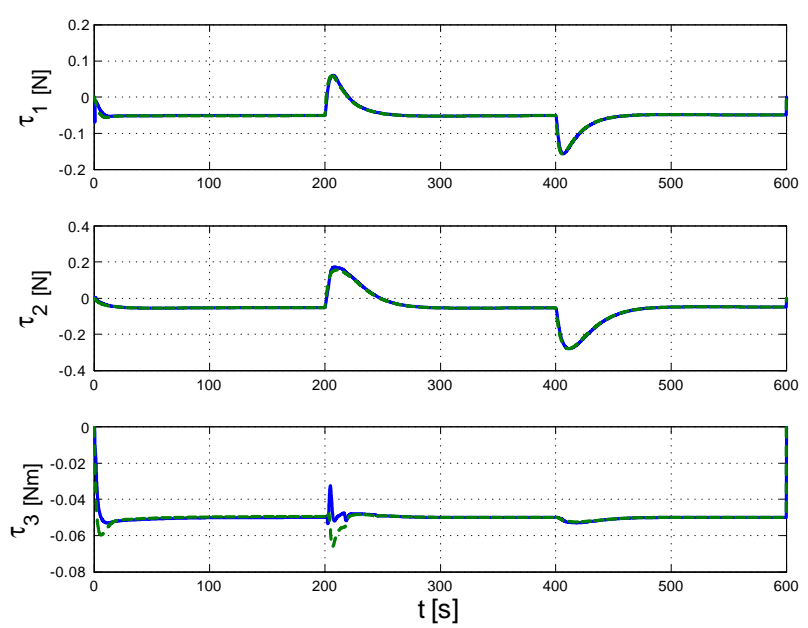

Fig. 7. Simulation results - the solid lines represent actual forces generated by the actuators, the dashed lines represent the virtual forces generated by the control law.

Enns, D. (1998), Control allocation approaches, in 'Proc. AIAA Guidance, Navigation and Control Conference and Exhibit, Boston MA', pp. 98-108.

Härkegård, O. (2002), Efficient active set algorithms for solving constrained least squares problems in aircraft control allocation, in 'Proc. IEEE Conf. Decision and Control, Las Vegas NV'.

Ioannou, P. A. \& Sun, J. (1996), Robust Adaptive Control, Prentice Hall PTR, New Jersey.

Johansen, T. A. (2004), 'Optimizing nonlinear control allocation', Proc. IEEE Conf. Decision and Control. Bahamas pp. 34353440 .

Johansen, T. A., Fossen, T. I. \& Berge, S. P. (2004), 'Constrained nonlinear control allocation with singularity avoidance using sequential quadratic programming', IEEE Trans. Control Systems Technology 12, 211-216.

Johansen, T. A., Fossen, T. I. \& Tøndel, P. (2005), 'Efficient optimal constrained control allocation via multi-parametric programming', AIAA J. Guidance, Control and Dynamics 28, 506-515.

Khalil, H. K. (1996), Nonlinear Systems, Prentice-Hall, Inc, New Jersey.

Krstic, M., Kanellakopoulos, I. \& Kokotovic, P. (1995), Nonlinear and Adaptive Control Design, John Wiley and Sons, Inc, New York.

Landau, H. (1979), Adaptive Control: The Model Reference Approach, Marcel Dekker, Inc., New York.

Lin, Y., Sontag, E. D. \& Wang, Y. (1996), 'A smooth converse lyapunov theorem for robust stability', SIAM Journal on Control and Optimization 34, 124-160.

Lindegaard, K. P. \& Fossen, T. I. (2003), 'Fuel-efficient rudder and propeller control allocation for marine craft: Experiments with a model ship', IEEE Trans. Control Systems Technology 11, 850-862.

Luo, Y., Serrani, A., Yurkovich, S., Doman, D. \& Oppenheimer, M. (2004), 'Model predictive dynamic control allocation with actuator dynamics', In Proceedings of the 2004 American Control Conference, Boston, MA .

Luo, Y., Serrani, A., Yurkovich, S., Doman, D. \& Oppenheimer, M. (2005), 'Dynamic control allocation with asymptotic tracking of time-varying control trajectories', In Proceedings of the 2005 American Control Conference, Portland, OR .

Mazenc, F. \& Praly, L. (1996), 'Adding integrations, saturated controls, and stabilization for feedforward systems', IEEE Transactions on Automatic Control 41, 1559-1578.

Nocedal, J. \& Wright, S. J. (1999), Numerical Optimization, Springer.

Pantely, E., Loria, A. \& Teel, A. R. (2001), 'Relaxed persistency of exitation for uniform asymptotic stability', IEEE Trans. Automat. Contr. 46, $1874-1886$.

Poonamallee, V., Yurkovich, S., Serrani, A., Doman, D. \& Oppenheimer, M. (2005), 'Dynamic control allocation with asymptotic tracking of time-varying control trajectories', In Proceedings of the 2004 American Control Conference, Boston, MA .

Skjetne, R. (2005), The Maneuvering Problem, Phd thesis, NTNU, Trondheim, Norway.

Sørdalen, O. J. (1997), 'Optimal thrust allocation for marine vessels', Control Engineering Practice 5, 1223-1231.

Teel, A., Panteley, E. \& Loria, A. (2002), 'Integral characterization of uniform asymptotic and exponential stability with applications', Maths. Control Signals and Systems 15, 177-201.

Tjønnås, J., Chaillet, A., Panteley, E. \& Johansen, T. A. (2006), 'Cascade lemma for set-stabile systems', 45th IEEE Conference on Decision and Control, San Diego, CA .

Tjønnås, J. \& Johansen, T. A. (2005), 'Adaptive optimizing nonlinear control allocation', In Proc. of the 16th IFAC World Congress, Prague, Czech Republic .

Tjønnås, J. \& Johansen, T. A. (2006), 'Adaptive optimizing dynamic control allocation algorithm for yaw stabilization of an automotive vehicle using brakes', 14 th Mediterranean Conference on Control and Automation, Ancona, Italy .

\section{A Proof of Lemma 2}

The boundary of the set $\mathcal{O}_{u \lambda}$ and the time-varying first order optimal solution is described by:

$$
\begin{aligned}
& \frac{\partial L}{\partial u}(t, x, u, \lambda, \theta)=\frac{\partial J}{\partial u}(t, x, u)-\left(\frac{\partial \Phi}{\partial u}(t, x, u, \theta)\right)^{\mathrm{T}} \lambda \\
& \frac{\partial L}{\partial \lambda}(t, x, u, \lambda, \theta)=k(t, x)-\Phi(t, x, u, \theta)
\end{aligned}
$$

By using theorem 2.4.7 in (Abrahamson, Marsden \& Ratiu 1988), it can be shown that

$$
\begin{aligned}
\frac{\partial L}{\partial u}(t, x, u, \lambda, \theta)-\frac{\partial L}{\partial u}\left(t, x, u_{c}, \lambda, \theta\right) & =\left.\frac{\partial^{2} L}{\partial u^{2}}\right|_{c}\left(u-u_{c}\right) \\
\frac{\partial L}{\partial u}\left(t, x, u_{c}, \lambda, \theta\right)-\frac{\partial L}{\partial u}\left(t, x, u_{c}, \lambda^{*}, \theta\right) & =\left.\frac{\partial^{2} L}{\partial \lambda \partial u}\right|_{c}\left(\lambda-\lambda^{*}\right) \\
\frac{\partial L}{\partial u}\left(t, x, u_{c}, \lambda^{*}, \theta\right)-\frac{\partial L}{\partial u}\left(t, x, u^{*}, \lambda^{*}, \theta\right) & =\left.\frac{\partial^{2} L}{\partial u^{2}}\right|_{*}\left(u_{c}-u^{*}\right) \\
\frac{\partial L}{\partial \lambda}(t, x, u, \lambda, \theta)-\frac{\partial L}{\partial \lambda}\left(t, x, u^{*}, \lambda, \theta\right) & =\left.\frac{\partial^{2} L}{\partial u \partial \lambda}\right|_{*}\left(u-u^{*}\right) \\
\frac{\partial L}{\partial \lambda}\left(t, x, u^{*}, \lambda, \theta\right)-\frac{\partial L}{\partial \lambda}\left(t, x, u^{*}, \lambda^{*}, \theta\right) & =0 .
\end{aligned}
$$


where $\left.\frac{\partial^{2} L}{\partial u^{2}}\right|_{c}:=\int_{0}^{1} \frac{\partial^{2} L}{\partial u^{2}}\left(t, x,(1-s) u_{c}+s u, \lambda, \theta\right) d s$, $\left.\frac{\partial^{2} L}{\partial u^{2}}\right|_{*}:=\int_{0}^{1} \frac{\partial^{2} L}{\partial u^{2}}\left(t, x,(1-s) u^{*}+s u_{c}, \lambda, \theta\right) d s,\left.\frac{\partial^{2} L}{\partial \lambda \partial u}\right|_{c}:=$ $\int_{0}^{1} \frac{\partial^{2} L}{\partial \lambda \partial u}\left(t, x, u_{c},(1-s) \lambda^{*}+s \lambda, \theta\right) d s=-\frac{\partial \Phi}{\partial u}^{\mathrm{T}}\left(t, x, u_{c}, \theta\right)$ and $\left.\frac{\partial^{2} L}{\partial u \partial \lambda}\right|_{*}:=\int_{0}^{1} \frac{\partial^{2} L}{\partial u \partial \lambda}\left(t, x,(1-s) u^{*}+s u, \lambda, \theta\right) d s=$ $-\frac{\partial \Phi}{\partial u}\left(t, x, u_{c}, \theta\right)$. Since

$\frac{\partial L}{\partial \lambda}\left(t, x, u^{*}, \lambda^{*}, \theta\right)=0$ and $\frac{\partial L}{\partial u}\left(t, x, u^{*}, \lambda^{*}, \theta\right)=0$, we get

$\frac{\partial L}{\partial u}(t, x, u, \lambda, \theta)=\left.\frac{\partial^{2} L}{\partial u^{2}}\right|_{c}\left(u-u_{c}\right)+\left.\frac{\partial^{2} L}{\partial u^{2}}\right|_{*}\left(u_{c}-u^{*}\right)$

$$
-\frac{\partial \Phi^{\mathrm{T}}}{\partial u}\left(t, x, u_{c}, \theta\right)\left(\lambda-\lambda^{*}\right)
$$

$\frac{\partial L}{\partial \lambda}(t, x, u, \lambda, \theta)=-\frac{\partial \Phi}{\partial u}\left(t, x, u_{c}, \theta\right)\left(u-u^{*}\right)$,

and from $u_{c}:=\vartheta\left(u-u^{*}\right)+u^{*}$ where $\vartheta:=\operatorname{diag}\left(\vartheta_{i}\right)$ and $0<\vartheta_{i}<1$ :

$$
\begin{aligned}
& \left.\frac{\partial^{2} L}{\partial u^{2}}\right|_{c}\left(u-u_{c}\right)+\left.\frac{\partial^{2} L}{\partial u^{2}}\right|_{*}\left(u_{c}-u^{*}\right) \\
= & \left.(1-\vartheta) \frac{\partial^{2} L}{\partial u^{2}}\right|_{c}\left(u-u^{*}\right)+\left.\vartheta \frac{\partial^{2} L}{\partial u^{2}}\right|_{*}\left(u-u^{*}\right) .
\end{aligned}
$$

From Assumption 3, $\left.\frac{\partial^{2} L}{\partial u^{2}}\right|_{c}$ and $\left.\frac{\partial^{2} L}{\partial u^{2}}\right|_{*}$ are positive definite matrices, such that $P_{* c}:=\left.(1-\vartheta) \frac{\partial^{2} L}{\partial u^{2}}\right|_{c}+\left.\vartheta \frac{\partial^{2} L}{\partial u^{2}}\right|_{*}$ is also a positive definite matrix. Further

$$
\left(\frac{\partial L}{\partial u}\right)^{\mathrm{T}} \frac{\partial L}{\partial u}+\left(\frac{\partial L}{\partial \lambda}\right)^{\mathrm{T}} \frac{\partial L}{\partial \lambda}=\left(\begin{array}{l}
u-u^{*} \\
\lambda-\lambda^{*}
\end{array}\right)^{\mathrm{T}} \mathbb{H}_{*}^{\mathrm{T}} \mathbb{H}_{*}\left(\begin{array}{l}
u-u^{*} \\
\lambda-\lambda^{*}
\end{array}\right)
$$

where $\mathbb{H}_{*}=\left(\begin{array}{cc}P_{* c} & \frac{\partial \Phi}{\partial u}^{\mathrm{T}}\left(t, x, u_{c}, \theta\right) \\ \frac{\partial \Phi}{\partial u}\left(t, x, u_{c}, \theta\right) & 0\end{array}\right)$. By Assumption 3 and 1 it can be seen that:

$$
\begin{aligned}
& \left|\operatorname{det}\left(\mathbb{H}_{*}\right)\right|= \\
& \left|\operatorname{det}\left(P_{* c}\right) \operatorname{det}\left(-\frac{\partial \Phi}{\partial u}\left(t, x, u_{c}, \theta\right) P_{* c}^{-1}\left(\frac{\partial \Phi}{\partial u}\left(t, x, u_{c}, \theta\right)\right)^{\mathrm{T}}\right)\right|
\end{aligned}
$$

and $\mathbb{H}_{*}$ is non-singular and there exists constants $\bar{\rho}_{2} \geq \bar{\rho}_{1}>0$ such that $\bar{\rho}_{1} I \leq \mathbb{H}_{*}^{\mathrm{T}} \mathbb{H}_{*} \bar{\rho}_{2} I$. Furthermore choose $u=0$ and $\lambda=0$, such that $\left|\frac{\partial L}{\partial u}(t, x, 0,0, \theta)\right| \leq \varsigma_{\partial J}(|x|)$ and $\left|\frac{\partial L}{\partial \lambda}(t, x, 0,0, \theta)\right| \leq$ $G_{\Phi}(|x|)(1+|\theta|)+\varsigma_{k}(|x|)$ from Assumption 1, 2 and 3.

Thus from (A.3) $\left|\left(u^{* \mathrm{~T}}, \lambda^{* \mathrm{~T}}\right)^{\mathrm{T}}\right| \leq \varsigma_{\mathcal{O} u \lambda}\left(\left|\left(x^{\mathrm{T}}, \theta^{\mathrm{T}}\right)^{\mathrm{T}}\right|\right)$ where $\varsigma_{\mathcal{O} u \lambda}(|s|):=\bar{\rho}_{1}^{-1}\left(G_{\Phi}(|s|)(1+|s|)+\varsigma_{k}(|s|)\right)^{2}+$ $\bar{\rho}_{1}^{-1} \varsigma_{\partial J}(|s|)^{2}$ Proof of Proposition 2

The proof is divided into two parts: $\mathbf{i}$ ) show that the integral of $\bar{f}_{\mathcal{O}_{u \lambda \tilde{\theta}}}(t)^{\mathrm{T}} \bar{f}_{\mathcal{O}_{u \lambda \tilde{\theta}}}(t)$, where

$$
\bar{f}_{\mathcal{O}_{u \lambda \tilde{\theta}}}(t):=f_{\mathcal{O}_{u \lambda \tilde{\theta}}}(t, x(t), u(t), \lambda(t), \epsilon(t), \tilde{\theta}(t)),
$$

is bounded, by using the PE property; and ii) use the integral bound to prove UGA by contradiction. The proof is based on ideas from (Pantely, Loria \& Teel 2001) and (Teel et al. 2002).

i) First we establish a bound on $\dot{\Phi}_{g}(t)$. We have

$$
\begin{aligned}
\dot{\Phi}_{g}(t)= & \frac{\partial g_{x}(t, x)}{\partial t} \Phi_{\theta}(t, x, u)+g_{x}(t, x) \frac{\partial \Phi_{\theta}(t, x, u)}{\partial t} \\
& +\left(\frac{\partial g_{x}(t, x)}{\partial x} \Phi_{\theta}(t, x, u)+g_{x}(t, x) \frac{\partial \Phi_{\theta}(t, x, u)}{\partial x}\right) \dot{x} \\
& +g_{x}(t, x) \frac{\partial \Phi_{\theta}(t, x, u)}{\partial u} \dot{u},
\end{aligned}
$$

thus from Assumption 1, system (28) and updatelaw (30), there exists a bound $\left|\dot{\Phi}_{g}(t)\right| \leq \varsigma_{1 x \dot{\Phi}_{g}}(|x|)+$ $\varsigma_{1 x \dot{\Phi}_{g}}(|x|) \varsigma_{u \dot{\Phi}_{g}}\left(\left|z_{u \lambda \tilde{\theta}}\right|_{\mathcal{O}_{u \lambda \tilde{\theta}}}\right)$ where $\varsigma_{1 x \dot{\Phi}_{g}}, \varsigma_{1 x \dot{\Phi}_{g}}, \varsigma_{1 x \dot{\Phi}_{g}}$ : $\mathbb{R}_{\geq 0} \rightarrow \mathbb{R}_{\geq 0}$ are continuous functions. This can be seen by following the same approach as in Lemma 3 . From the assumption that $x$ is uniformly bounded, we use $|x| \leq B_{x}$. The integrability of $\tilde{\theta}^{\mathrm{T}} \tilde{\theta}$ is investigated by considering the auxiliary function: $V_{\theta a u x 1}:=$ $-\epsilon^{\mathrm{T}} \Phi_{g}(t) \tilde{\theta}$, bounded by $B_{\text {V } \operatorname{aux} 1}\left(B_{x}, r\right) \geq V_{\text {a aux } 1}$ where $r \geq\left|z_{u \lambda \tilde{\theta} 0}\right|_{\mathcal{O}_{u \lambda \tilde{\theta}}}$ and $r>0$. Its derivative along the solutions of (27) and (31) is given by

$$
\begin{aligned}
\dot{V}_{\text {taux } 1}= & -\tilde{\theta}^{\mathrm{T}} \Phi_{g}(t)^{\mathrm{T}} \Phi_{g}(t) \tilde{\theta}+\epsilon^{\mathrm{T}}\left(A^{\mathrm{T}} \Phi_{g}(t)-\dot{\Phi}_{g}(t)\right) \tilde{\theta} \\
& +\epsilon^{\mathrm{T}} \Phi_{g}(t) \Gamma_{\hat{\theta}}^{-1} \Phi_{g}(t)^{\mathrm{T}} \epsilon \\
& +\epsilon^{\mathrm{T}} \Phi_{g}(t) \Gamma_{\hat{\theta}}^{-1} \Phi_{g}(t)^{\mathrm{T}} \frac{\partial_{x}^{2} \bar{L}_{\hat{\theta}}}{\partial u \lambda}(t) \frac{\partial \bar{L}_{\hat{\theta}}}{\partial u \lambda}(t)
\end{aligned}
$$

where $\frac{\partial_{x}^{2} \bar{L}_{\hat{\theta}}}{\partial u \lambda}(t):=\left(\left(\frac{\partial^{2} L_{\hat{\theta}}}{\partial x \partial u}\right)^{\mathrm{T}},\left(\frac{\partial^{2} L_{\hat{\theta}}}{\partial x \partial \lambda}\right)^{\mathrm{T}}\right)(t, x(t), u(t), \lambda(t), \hat{\theta}(t))$ and $\frac{\partial \bar{L}_{\hat{\theta}}}{\partial u \lambda}(t):=\left(\left(\frac{\partial L_{\hat{\theta}}}{\partial u}\right)^{\mathrm{T}}\left(\frac{\partial L_{\hat{\theta}}}{\partial \lambda}\right)^{\mathrm{T}}\right)^{\mathrm{T}}(t, x(t), u(t), \lambda(t), \hat{\theta}(t))$. From Young's inequality, $n a^{\mathrm{T}} a+\frac{1}{n} b^{\mathrm{T}} b \geq 2\left|b^{\mathrm{T}} a\right|$, we have

$$
\begin{aligned}
& \epsilon^{\mathrm{T}}\left(A^{\mathrm{T}} \Phi_{g}(t)-\dot{\Phi}_{g}(t)\right) \tilde{\theta} \\
\leq & \frac{T}{\mu} \epsilon^{\mathrm{T}}\left(A^{\mathrm{T}} \Phi_{g}(t)-\dot{\Phi}_{g}(t)\right)\left(A^{\mathrm{T}} \Phi_{g}(t)-\dot{\Phi}_{g}(t)\right)^{\mathrm{T}} \epsilon \\
& +\frac{\mu}{4 T} \tilde{\theta}^{\mathrm{T}} \tilde{\theta},
\end{aligned}
$$

where $a=\tilde{\theta}, b=\epsilon^{\mathrm{T}}\left(A^{\mathrm{T}} \Phi_{g}(t)-\dot{\Phi}_{g}(t)\right)$ and $n=\frac{\mu}{2 T}$ such that $\mu$ and $T$ are positive scalars of desirable choice. Next

$$
\begin{aligned}
& \epsilon^{\mathrm{T}} \Phi_{g}(t) \Gamma_{\hat{\theta}}^{-1} \Phi_{g}(t)^{\mathrm{T}} \frac{\partial_{x}^{2} \bar{L}_{\hat{\theta}}}{\partial u \lambda}(t) \frac{\partial \bar{L}_{\hat{\theta}}}{\partial u \lambda}(t) \\
\leq & \epsilon^{\mathrm{T}} \Phi_{g}(t) \Phi_{g}^{\mathrm{T}}(t) \epsilon+\left(\frac{\partial \bar{L}_{\hat{\theta}}}{\partial u \lambda}(t)\right)^{\mathrm{T}} K_{L \Phi} \frac{\partial \bar{L}_{\hat{\theta}}}{\partial u \lambda}(t)
\end{aligned}
$$


for $n=1, a=\epsilon^{\mathrm{T}} \Phi_{g}(t)$ and $b=\Gamma_{\hat{\theta}}^{-1} \Phi_{g}(t)^{\mathrm{T}} \frac{\partial_{x}^{2} \bar{L}_{\hat{\theta}}}{\partial u \lambda}(t) \frac{\partial \bar{L}_{\hat{\theta}}}{\partial u \lambda}(t)$, where $K_{L \Phi}:=\frac{\partial_{x}^{2} \bar{L}_{\hat{\theta}}}{\partial u \lambda}(t) \Phi_{g}(t) \Gamma_{\hat{\theta}}^{-\mathrm{T}} \Gamma_{\hat{\theta}}^{-1} \Phi_{g}^{\mathrm{T}}(t) \frac{\partial_{x}^{2} \bar{L}_{\hat{\theta}}^{\mathrm{T}}}{\partial u \lambda}(t)$. Hence

$$
\begin{aligned}
\dot{V}_{\text {taux } 1} \leq & \frac{T}{\mu} \epsilon^{\mathrm{T}}\left(A^{\mathrm{T}} \Phi_{g}(t)-\dot{\Phi}_{g}(t)\right)\left(A^{\mathrm{T}} \Phi_{g}(t)-\dot{\Phi}_{g}(t)\right)^{\mathrm{T}} \epsilon \\
& +\epsilon^{\mathrm{T}} \Phi_{g}(t) \Gamma_{\hat{\theta}}^{-1} \Phi_{g}(t)^{\mathrm{T}} \epsilon-\tilde{\theta}^{\mathrm{T}} \Phi_{g}(t)^{\mathrm{T}} \Phi_{g}(t) \tilde{\theta} \\
& +\frac{\mu}{4 T} \tilde{\theta}^{\mathrm{T}} \tilde{\theta}+\epsilon^{\mathrm{T}} \Phi_{g}(t) \Phi_{g}^{\mathrm{T}}(t) \epsilon \\
& +\frac{\partial \bar{L}_{\hat{\theta}}^{\mathrm{T}}}{\partial u \lambda}(t) K_{L \Phi} \frac{\partial \bar{L}_{\hat{\theta}}}{\partial u \lambda}(t),
\end{aligned}
$$

and thus

$$
\begin{aligned}
& \tilde{\theta}(t)^{\mathrm{T}}\left(\Phi_{g}(t)^{\mathrm{T}} \Phi_{g}(t)-\frac{\mu}{4 T} I\right) \tilde{\theta}(t) \\
\leq & \frac{T}{\mu} \epsilon^{\mathrm{T}}\left(A^{\mathrm{T}} \Phi_{g}(t)-\dot{\Phi}_{g}(t)\right)\left(A^{\mathrm{T}} \Phi_{g}(t)-\dot{\Phi}_{g}(t)\right)^{\mathrm{T}} \epsilon \\
& +\epsilon^{\mathrm{T}} \Phi_{g}(t)\left(\Gamma_{\hat{\theta}}^{-1}+I\right) \Phi_{g}^{\mathrm{T}}(t) \epsilon \\
& -\dot{V}_{\text {சaux } 1}+\frac{\partial \bar{L}_{\hat{\theta}}^{\mathrm{T}}}{\partial u \lambda}(t) K_{L \Phi} \frac{\partial \bar{L}_{\hat{\theta}}}{\partial u \lambda}(t) .
\end{aligned}
$$

The solution of (30) can be represented by: $\tilde{\theta}(\tau)=$ $\tilde{\theta}(t)-R_{\Phi}(t, \tau)$, where

$R_{\Phi}(t, \tau):=\Gamma_{\hat{\theta}}^{-1} \int_{t}^{\tau} \Phi_{g}^{\mathrm{T}}(s)\left(\epsilon(s)+\frac{\partial_{x}^{2} \bar{L}_{\hat{\hat{\lambda}}}}{\partial u \lambda}(s) \frac{\partial \bar{L}_{\hat{\theta}}}{\partial u \lambda}(s)\right) d s$, such that

$$
\begin{aligned}
& \tilde{\theta}(\tau)^{\mathrm{T}}\left(\Phi_{g}(\tau)^{\mathrm{T}} \Phi_{g}(\tau)-\frac{\mu}{4 T} I\right) \tilde{\theta}(\tau) \\
= & \tilde{\theta}(t)^{\mathrm{T}}\left(\Phi_{g}(\tau)^{\mathrm{T}} \Phi_{g}(\tau)-\frac{\mu}{4 T} I\right) \tilde{\theta}(t) \\
& +R_{\Phi}(t, \tau)^{\mathrm{T}}\left(\Phi_{g}(\tau)^{\mathrm{T}} \Phi_{g}(\tau)-\frac{\mu}{4 T} I\right) R_{\Phi}(t, \tau) \\
& -2 \tilde{\theta}(t)^{\mathrm{T}}\left(\Phi_{g}(\tau)^{\mathrm{T}} \Phi_{g}(\tau)-\frac{\mu}{4 T} I\right) R_{\Phi}(t, \tau) .
\end{aligned}
$$

Again by Young's inequality, $-n_{1} a_{1}^{\mathrm{T}} a_{1}-\frac{1}{n_{1}} b_{1}^{\mathrm{T}} b_{1} \leq$ $-2\left|b_{1}^{\mathrm{T}} a_{1}\right|$ and $-n_{2} a_{2}^{\mathrm{T}} a_{2}-\frac{1}{n_{2}} b_{2}^{\mathrm{T}} b_{2} \leq 2\left|b_{2}^{\mathrm{T}} a_{2}\right|$ with $a_{1}=\Phi_{g}(\tau) \tilde{\theta}, a_{2}=\sqrt{\frac{\mu}{4 T}} \tilde{\theta}, b_{1}=\Phi_{g}(\tau) R_{\Phi}(t, \tau)$, $b_{2}=\sqrt{\frac{\mu}{4 T}} R_{\Phi}(t, \tau)$ and $n_{1}=n_{2}=\frac{1}{\sigma}$, such that

$$
-2 \tilde{\theta}(t)^{\mathrm{T}} \Phi_{g}(\tau)^{\mathrm{T}} \Phi_{g}(\tau) R_{\Phi}(t, \tau) \geq-\frac{1}{\sigma} \tilde{\theta}(t)^{\mathrm{T}} \Phi_{g}(\tau)^{\mathrm{T}} \Phi_{g}(\tau) \tilde{\theta}(t)
$$$$
-\sigma R_{\Phi}(t, \tau)^{\mathrm{T}} \Phi_{g}(\tau)^{\mathrm{T}} \Phi_{g}(\tau) R_{\Phi}(t, \tau)
$$

and

$$
\begin{aligned}
& 2 \frac{\mu}{4 T} \tilde{\theta}(t)^{\mathrm{T}} R_{\Phi}(t, \tau) \geq-\frac{1}{\sigma} \frac{\mu}{4 T} \tilde{\theta}(t)^{\mathrm{T}} \Phi_{g}(\tau)^{\mathrm{T}} \Phi_{g}(\tau) \tilde{\theta}(t) \\
& -\sigma \frac{\mu}{4 T} R_{\Phi}(t, \tau)^{\mathrm{T}} \Phi_{g}(\tau)^{\mathrm{T}} \Phi_{g}(\tau) R_{\Phi}(t, \tau)
\end{aligned}
$$

which gives

$$
\begin{aligned}
& \tilde{\theta}(\tau)^{\mathrm{T}}\left(\Phi_{g}(\tau)^{\mathrm{T}} \Phi_{g}(\tau)-\frac{\mu}{4 T} I\right) \tilde{\theta}(\tau) \\
& \geq(1-\sigma) R_{\Phi}(t, \tau)^{\mathrm{T}}\left(\Phi_{g}(\tau)^{\mathrm{T}} \Phi_{g}(\tau)-\frac{(1+\sigma) \mu}{(1-\sigma) 4 T} I\right) R_{\Phi}(t, \tau) \\
& +\left(1-\frac{1}{\sigma}\right) \tilde{\theta}(t)^{\mathrm{T}}\left(\Phi_{g}(\tau)^{\mathrm{T}} \Phi_{g}(\tau)-\frac{\left(1+\frac{1}{\sigma}\right)}{\left(1-\frac{1}{\sigma}\right)} \frac{\mu}{4 T} I\right) \tilde{\theta}(t)(\text { B. } 7)
\end{aligned}
$$

for $\sigma>1$. From Holder's inequality,

$\int|f g| \leq\left(\int|f|^{2}\right)^{\frac{1}{2}}\left(\int|g|^{2}\right)^{\frac{1}{2}}$, with $g=1$ and $f=$ $\Gamma_{\hat{\theta}}^{-1} \Phi_{g}^{\mathrm{T}}(s) \epsilon(s)$ we get

$\left(\int_{t}^{\tau}\left|\Gamma_{\hat{\theta}}^{-1} \Phi_{g}^{\mathrm{T}}(s) \epsilon(s)\right| d s\right)^{2} \leq \int_{t}^{\tau}\left|\Gamma_{\hat{\theta}}^{-1} \Phi_{g}^{\mathrm{T}}(s) \epsilon(s)\right|^{2} d s(\tau-t)$

and by similar arguments

$$
\begin{aligned}
& \left(\int_{t}^{\tau}\left|\Gamma_{\hat{\theta}}^{-1} \Phi_{g}^{\mathrm{T}}(s) \frac{\partial_{x}^{2} \bar{L}_{\hat{\theta}}}{\partial u \lambda}(s) \frac{\partial \bar{L}_{\hat{\theta}}}{\partial u \lambda}(s)\right| d s\right)^{2} \\
\leq & \int_{t}^{\tau}\left|\Gamma_{\hat{\theta}}^{-1} \Phi_{g}^{\mathrm{T}}(s) \frac{\partial_{x}^{2} \bar{L}_{\hat{\theta}}}{\partial u \lambda}(s) \frac{\partial \bar{L}_{\hat{\theta}}}{\partial u \lambda}(s)\right|^{2} d s(\tau-t),
\end{aligned}
$$

such that

$$
\begin{aligned}
& R_{\Phi}(t, \tau)^{\mathrm{T}} R_{\Phi}(t, \tau) \\
\leq & 2 \int_{t}^{\tau} \epsilon(s)^{\mathrm{T}} \Phi_{g}(s) \Gamma_{\hat{\theta}}^{-\mathrm{T}} \Gamma_{\hat{\theta}}^{-1} \Phi_{g}^{\mathrm{T}}(s) \epsilon(s) d s(\tau-t) \\
& +2 \int_{t}^{\tau} \frac{\partial \bar{L}_{\hat{\theta}}^{\mathrm{T}}}{\partial u \lambda}(s) K_{L \Phi} \frac{\partial \bar{L}_{\hat{\theta}}}{\partial u \lambda}(s) d s(\tau-t) .
\end{aligned}
$$

Let $B_{\Phi T}\left(B_{x}, r, T\right):=\lambda_{\max }\left(\Phi_{g}(t)^{\mathrm{T}} \Phi_{g}(t)-\frac{(1+\sigma)}{(1-\sigma)} \frac{\mu}{4 T} I\right)$ be the maximal eigenvalue of $\left(\Phi_{g}(\tau)^{\mathrm{T}} \Phi_{g}(\tau)-\frac{(1+\sigma)}{(1-\sigma)} \frac{\mu}{4 T} I\right)$ and $\lambda_{\max } K_{L \Phi}$ be the maximal eigenvalue of $K_{L \Phi}$, then from combining (B.5) and (B.7) and investigating the integral over $\tau$, we get

$$
\begin{aligned}
& \left(1-\frac{1}{\sigma}\right) \tilde{\theta}(t)^{\mathrm{T}} \int_{t}^{t+T}\left(\Phi_{g}(\tau)^{\mathrm{T}} \Phi_{g}(\tau)-\frac{\left(1+\frac{1}{\sigma}\right)}{\left(1-\frac{1}{\sigma}\right)} \frac{\mu}{4 T} I\right) d \tau \tilde{\theta}(t) \\
& \leq B_{\Phi T} \int_{t}^{t+T}\left(\int_{t}^{\tau} \epsilon(s)^{\mathrm{T}} \Phi_{g}(s) \Phi_{g}^{\mathrm{T}}(s) \epsilon(s) d s\right)(\tau-t) d \tau \\
& +B_{\Phi T} \lambda_{\max } K_{L \Phi} \int_{t}^{t+T}\left(\int_{t}^{\tau} \frac{\partial \bar{L}_{\hat{\theta}}^{\mathrm{T}}}{\partial u \lambda}(s)^{\mathrm{T}} \frac{\partial \bar{L}_{\hat{\theta}}}{\partial u \lambda}(s) d s\right)(\tau-t) d \tau \\
& +V_{\theta a u x 1}(t)-V_{\theta a u x 1}(t+T) \\
& +\lambda_{\max } K_{L \Phi} \int_{t}^{t+T}\left(\frac{\partial \bar{L}_{\hat{\theta}}^{\mathrm{T}}}{\partial u \lambda}(s) \frac{\partial \bar{L}_{\hat{\theta}}}{\partial u \lambda}(s)\right) d s \\
& +\int_{t}^{t+T} \epsilon(\tau)^{\mathrm{T}} \Phi_{g}(\tau)\left(\Gamma_{\hat{\theta}}^{-1}+I\right) \Phi_{g}^{\mathrm{T}}(\tau) \epsilon(\tau) d \tau \\
& +\frac{T}{\mu} \int_{t}^{t+T} \epsilon(\tau)^{\mathrm{T}}\left(A^{\mathrm{T}} \Phi_{g}(\tau)-\dot{\Phi}_{g}(\tau)\right)\left(A^{\mathrm{T}} \Phi_{g}(\tau)-\dot{\Phi}_{g}(\tau)\right)^{\mathrm{T}} \epsilon(\tau) d \tau .
\end{aligned}
$$

Further 


$$
\begin{aligned}
& \int_{t}^{t+T}\left(\int_{t}^{\tau} \epsilon(s)^{\mathrm{T}} \Phi_{g}(s) \Gamma_{\hat{\theta}}^{-\mathrm{T}} \Gamma_{\hat{\theta}}^{-1} \Phi_{g}^{\mathrm{T}}(s) \epsilon(s) d s\right)(\tau-t) d \tau \\
\leq & T^{2} \int_{t}^{t+T} \epsilon(s)^{\mathrm{T}} \Phi_{g}(s) \Gamma_{\hat{\theta}}^{-\mathrm{T}} \Gamma_{\hat{\theta}}^{-1} \Phi_{g}^{\mathrm{T}}(s) \epsilon(s) d s,
\end{aligned}
$$

such that for

$$
\begin{aligned}
& B_{\epsilon}\left(B_{x}, r, T\right) \\
:= & \lambda_{\max }\left(\left(A^{\mathrm{T}} \Phi_{g}(t)-\dot{\Phi}_{g}(t)\right)\left(A^{\mathrm{T}} \Phi_{g}(t)-\dot{\Phi}_{g}(t)\right)^{\mathrm{T}}+\right. \\
& \left.\Phi_{g}(t)\left(\left(B_{\Phi T} T^{2}+1\right) \Gamma_{\hat{\theta}}^{-1}+I\right) \Phi_{g}(t)^{\mathrm{T}}\right)
\end{aligned}
$$

it follows that

$$
\begin{aligned}
& \left(1-\frac{1}{\sigma}\right) \tilde{\theta}(t)^{\mathrm{T}} \int_{t}^{t+T}\left(\Phi_{g}(\tau)^{\mathrm{T}} \Phi_{g}(\tau)-\frac{\left(1+\frac{1}{\sigma}\right)}{\left(1-\frac{1}{\sigma}\right)} \frac{\mu}{4 T} I\right) d \tau \tilde{\theta}(t) \\
& \leq V_{\theta a u x 1}(t)-V_{\theta a u x 1}(t+T) \\
& \left.+B_{V} \int_{t}^{t+T}\left(\left(\frac{\partial \bar{L}_{\hat{\theta}}}{\partial u \lambda}(\tau)\right)\right)^{\mathrm{T}} \mathbb{H}_{\hat{\theta}}^{\mathrm{T}}(\tau) \Gamma \mathbb{H}_{\hat{\theta}}(\tau) \frac{\partial \bar{L}_{\hat{\theta}}}{\partial u \lambda}(\tau)+\epsilon(\tau)^{\mathrm{T}} A \epsilon(\tau)\right) d \tau
\end{aligned}
$$

and

$$
\begin{aligned}
& \tilde{\theta}(t)^{\mathrm{T}} \int_{t}^{t+T}\left(\Phi_{g}(\tau)^{\mathrm{T}} \Phi_{g}(\tau)-\frac{\left(1+\frac{1}{\sigma}\right)}{\left(1-\frac{1}{\sigma}\right)} \frac{\mu}{4 T} I\right) d \tau \tilde{\theta}(t) \\
\leq & \frac{1}{\left(1-\frac{1}{\sigma}\right)}\left(V_{\theta a u x 1}(t)-V_{\theta a u x 1}(t+T)\right) \\
& +B_{V}\left(V_{u \lambda \hat{\theta}}(T+t)-V_{u \lambda \hat{\theta}}(t)\right),
\end{aligned}
$$

where $B_{V}\left(B_{x}, r, T\right):=\frac{\lambda_{\max } B_{\epsilon}(t, T)+\lambda_{\max } K_{L \Phi}\left(B_{\Phi T} T^{2}+1\right)}{\left(1-\frac{1}{\sigma}\right) \lambda_{\min } A}$. By the PE assumption we can choose $T, \gamma, \mu>0$ and $\sigma>1$ such that, $B_{\gamma}<\gamma-\frac{\left(1+\frac{1}{\sigma}\right)}{\left(1-\frac{1}{\sigma}\right)} \frac{\mu}{4}$, where $B_{\gamma}>0$ i.e.

$$
\begin{aligned}
& B_{\gamma} \tilde{\theta}(t)^{\mathrm{T}} \tilde{\theta}(t) \\
& \leq \tilde{\theta}(t)^{\mathrm{T}} \int_{t}^{t+T}\left(\Phi_{g}(\tau)^{\mathrm{T}} \Phi_{g}(\tau)-\frac{\left(1+\frac{1}{\sigma}\right)}{\left(1-\frac{1}{\sigma}\right)} \frac{\mu}{4 T} I\right) d \tau \tilde{\theta}(t)
\end{aligned}
$$

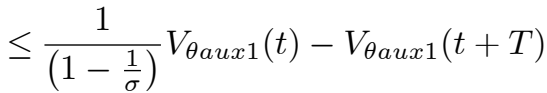

$$
\begin{aligned}
& +B_{V}\left(V_{u \lambda \hat{\theta}}(T+t)-V_{u \lambda \hat{\theta}}(t)\right) \text {, }
\end{aligned}
$$

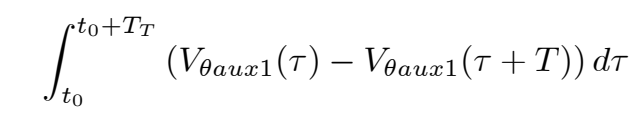$$
=\int_{t_{0}}^{t_{0}+T} V_{\theta a u x 1}(\tau) d \tau-\int_{t_{0}+T_{T}}^{t_{0}+T_{T}+T} V_{\theta a u x 1}(\tau) d \tau
$$$$
\leq 2 T B_{V \operatorname{\theta aux} 1}\left(B_{x}, r\right)
$$

and

$$
\int_{t_{0}}^{t_{0}+T_{T}} \tilde{\theta}(\tau)^{\mathrm{T}} \tilde{\theta}(\tau) d \tau \leq \frac{2 T}{B_{\gamma}}\left(\frac{B_{V \theta a u x 1}\left(B_{x}, r\right)}{\left(1-\frac{1}{\sigma}\right)}+B_{V} V_{u \lambda \hat{\theta}}\left(t_{0}\right)\right) .
$$

Thus $\tilde{\theta}(\tau)^{\mathrm{T}} \tilde{\theta}(\tau)$ is integrable. Let $\lambda_{\min } V$ be the minimum eigenvalue of $\left(1, \mathbb{H}_{\hat{\theta}}^{\mathrm{T}} \Gamma \mathbb{H}_{\hat{\theta}}, A\right)$, then there exists a constant $B_{f_{\mathcal{O}}{ }_{u \lambda \tilde{\theta}}}\left(B_{x}, r, T\right)>0$, such that

$$
\begin{aligned}
& \int_{t_{0}}^{t_{0}+T_{T}} \bar{f}_{\mathcal{O}_{u \lambda \tilde{\theta}}}(\tau)^{\mathrm{T}} \bar{f}_{\mathcal{O}_{u \lambda \tilde{\theta}}}(\tau) d \tau \\
\leq & \lim _{t \rightarrow \infty} \int_{t_{0}}^{t} \bar{f}_{\mathcal{O}_{u \lambda \tilde{\theta}}}(\tau)^{\mathrm{T}} \bar{f}_{\mathcal{O}_{u \lambda \tilde{\theta}}}(\tau) d \tau \\
\leq & \frac{1}{\lambda_{\min } V}\left(V_{u \lambda \hat{\theta}}\left(t_{0}\right)-V_{u \lambda \hat{\theta}}(\infty)\right) \\
& +\frac{2 T}{B_{\gamma}}\left(\frac{B_{V \theta a u x 1}\left(B_{x}, r\right)}{\left(1-\frac{1}{\sigma}\right)}+B_{V} V_{u \lambda \hat{\theta}}\left(t_{0}\right)\right) \\
\leq & \left.B_{f_{\mathcal{O}}}\right)
\end{aligned}
$$

and the integral of $\bar{f}_{\mathcal{O}_{u \lambda \tilde{\theta}}}(t)^{\mathrm{T}} \bar{f}_{\mathcal{O}_{u \lambda \tilde{\theta}}}(t)$ is bounded.

ii) From The UGS property we have

$$
\left|z_{u \lambda \tilde{\theta}}(t)\right|_{\mathcal{O}_{u \lambda \tilde{\theta}}} \leq \rho\left(\left|z_{u \lambda \tilde{\theta} 0}\right|_{\mathcal{O}_{u \lambda \tilde{\theta}}}\right), \quad \forall t \geq t_{0}
$$

where $\rho \in \mathcal{K}_{\infty}$. Fix $r>0, \varepsilon>0$. Define $\Omega:=\rho(r)$ and

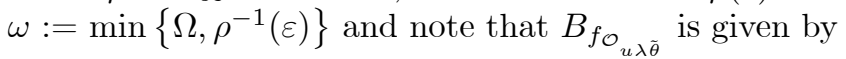
$\omega$ and $\Omega$. Define $T_{T}=\frac{2 B_{f_{\mathcal{O}}}}{\omega_{1}}$, where $\omega_{1}$ is specified later, and assume that for all $\left|z_{u \lambda \tilde{\theta} 0}\right|_{\mathcal{O}_{u \lambda \tilde{\theta}}} \leq r$, there exists $t^{\prime} \in\left[t_{0}, T_{T}\right]$ such that $\left|z_{u \lambda \tilde{\theta}}\left(t^{\prime}, z_{u \lambda \tilde{\theta} 0}\right)\right|_{\mathcal{A}} \leq \rho^{-1}(\varepsilon)$. Thus $\left|z_{u \lambda \tilde{\theta}}\left(t, z_{u \lambda \tilde{\theta} 0}\right)\right|_{\mathcal{O}_{u \lambda \tilde{\theta}}} \leq \rho\left(\left|z_{u \lambda \tilde{\theta}}\left(t^{\prime}, z_{u \lambda \tilde{\theta} 0}\right)\right|_{\mathcal{O}_{u \lambda \tilde{\theta}}}\right) \leq$ $\rho\left(\rho^{-1}(\varepsilon)\right)=\varepsilon$ for $\left|z_{u \lambda \tilde{\theta} 0}\right|_{\mathcal{O}_{u \lambda \tilde{\theta}}} \leq r$ and $t \geq T_{T}+t_{0}$, which satisfies definition 3. Suppose the assumption is not true, i.e., there exists $\left|z_{u \lambda \tilde{\theta} 0}\right|_{\mathcal{O}_{u \lambda \tilde{\theta}}} \leq r$ such that $\left|z_{u \lambda \tilde{\theta}}\left(t^{\prime}, z_{u \lambda \tilde{\theta} 0}\right)\right|_{\mathcal{O}_{u \lambda \tilde{\theta}}}>\rho^{-1}(\varepsilon) \forall t^{\prime} \in\left[t_{0}, T_{T}\right]$. Thus

$$
\omega \leq\left|z_{u \lambda \tilde{\theta}}\left(t^{\prime}, z_{u \lambda \tilde{\theta} 0}\right)\right|_{\mathcal{O}_{u \lambda \tilde{\theta}}} \leq \Omega, \quad \forall t^{\prime} \in\left[t_{0}, T_{T}\right]
$$

which from radial unboundedness of $V_{u \lambda \hat{\theta}}$, related to (33), imply that there exist positive constants $\omega_{1}$, and $\Omega_{1}$ such that

$$
\omega_{1} \leq \bar{f}_{\mathcal{O}_{u \lambda \tilde{\theta}}}\left(t^{\prime}\right)^{\mathrm{T}} \bar{f}_{\mathcal{O}_{u \lambda \tilde{\theta}}}\left(t^{\prime}\right) \leq \Omega_{1}, \quad \forall t^{\prime} \in\left[t_{0}, T_{T}\right]
$$

Then

$$
\begin{aligned}
& \int_{t_{0}}^{t_{0}+T_{T}} \bar{f}_{\mathcal{O}_{u \lambda \tilde{\theta}}}(\tau)^{\mathrm{T}} \bar{f}_{\mathcal{O}_{u \lambda \tilde{\theta}}}(\tau) d \tau \geq \int_{t_{0}}^{t_{0}+T_{T}} \omega_{1} d \tau \\
= & \omega_{1} T_{T} \\
= & 2 B_{f_{\mathcal{O}_{u \lambda \tilde{\theta}}}}
\end{aligned}
$$

which contradicts (B.8), and the proposition is proved. 